\title{
Identification of the specific long- noncoding RNAs involved in night-break mediated flowering retardation in Chenopodium quinoa
}

Qi Wu*, Yiming Luo, Xiaoyong Wu, Xue Bai, Xueling Ye, Changying Liu, Yan Wan, Dabing Xiang, Qiang Li, Liang Zou and Gang Zhao

\begin{abstract}
Background: Night-break (NB) has been proven to repress flowering of short-day plants (SDPs). Long-noncoding RNAs (IncRNAs) play key roles in plant flowering. However, investigation of the relationship between IncRNAs and NB responses is still limited, especially in Chenopodium quinoa, an important short-day coarse cereal.

Results: In this study, we performed strand-specific RNA-seq of leaf samples collected from quinoa seedlings treated by SD and NB. A total of 4914 high-confidence IncRNAs were identified, out of which 91 IncRNAs showed specific responses to SD and NB. Based on the expression profiles, we identified 17 positive- and 7 negativeflowering IncRNAs. Co-expression network analysis indicated that 1653 mRNAs were the common targets of both types of flowering IncRNAs. By mapping these targets to the known flowering pathways in model plants, we found some pivotal flowering homologs, including 2 florigen encoding genes (FT (FLOWERING LOCUS T) and TSF (TWIN SISTER of FT) homologs), 3 circadian clock related genes (EARLY FLOWERING 3 (ELF3), LATE ELONGATED HYPOCOTYL (LHY) and ELONGATED HYPOCOTYL 5 (HY5) homologs), 2 photoreceptor genes (PHYTOCHROME A (PHYA) and CRYP TOCHROME1 (CRY1) homologs), 1 B-BOX type CONSTANS (CO) homolog and 1 RELATED TO ABIBNP1 (RAV1) homolog, were specifically affected by NB and competed by the positive and negative-flowering IncRNAs. We speculated that these potential flowering IncRNAs may mediate quinoa NB responses by modifying the expression of the floral homologous genes.
\end{abstract}

Conclusions: Together, the findings in this study will deepen our understanding of the roles of IncRNAs in NB responses, and provide valuable information for functional characterization in future.

Keywords: LncRNA, Night break, Flowering, Chenopodium quinoa, Co-expression network

\footnotetext{
*Correspondence: jerviswuqi@126.com

Key Laboratory of Coarse Cereal Processing, Ministry of Agriculture and Rural Affairs, Sichuan Engineering \& Technology Research Center of Coarse Cereal Industralization, School of Food and Biological Engineering, Chengdu University, Chengluo road 2025, Shiling town, Longquanyi District, Chengdu 610106, Sichuan Province, P.R. China
}

(c) The Author(s). 2021 Open Access This article is licensed under a Creative Commons Attribution 4.0 International License, which permits use, sharing, adaptation, distribution and reproduction in any medium or format, as long as you give appropriate credit to the original author(s) and the source, provide a link to the Creative Commons licence, and indicate if changes were made. The images or other third party material in this article are included in the article's Creative Commons licence, unless indicated otherwise in a credit line to the material. If material is not included in the article's Creative Commons licence and your intended use is not permitted by statutory regulation or exceeds the permitted use, you will need to obtain permission directly from the copyright holder. To view a copy of this licence, visit http://creativecommons.org/licenses/by/4.0/ The Creative Commons Public Domain Dedication waiver (http://creativecommons.org/publicdomain/zero/1.0/) applies to the data made available in this article, unless otherwise stated in a credit line to the data. 


\section{Background}

Plants sense environmental changes to maximize flowering transition success. Accumulating evidences have revealed that six major factors, including temperature, photoperiod, gibberellin acids (GAs), age and autonomous pathways are implicated in flowering regulation [1]. The photoperiod pathway controls floral transition through a signal cascade involving circadian clock and florigen genes. Under inductive photoperiods when the expression phases of endogenous circadian clock regulators coincide with external light signal, the clock output genes are converged to activate the florigen-encoding genes, FLOWERING LOCUS $T(F T)$ and its homologs [2-4]. Thereafter, FT proteins are transported to shoot apical meristems where interact with FLOWERING LOCUS T (FD) and 14-3-3 proteins to form florigen activation complex (FAC), which in turn induces the meristem identity gene APETALA1 and triggers flowering $[5,6]$. Day length is a critical factor for photoperiodic flowering. In short-day plants (SDPs) such as rice (Oryza saltiva) and soybean (Glycine max), FT transcripts are more abundant when the night length longer than a certain threshold [7, 8]. By contrast, in long-day plants (LDPs) such as Arabidopsis (Arabidopsis thaliana) and wheat (Triticum aestivum), only when the day length exceeds a certain threshold, FT proteins are sufficient for flowering induction [9, 10]. Different photoperiods combined with short pulses of light during the night period (referred to as night break, NB) approaches are well-established and widely adopted to study the photoperiodic regulation of flowering in many plant species. NB causes different effects in flowering of SDPs and LDPs by changing the expression of FT. In rice, Heading date $3 a(H d 3 a)$, a homo$\log$ of Arabidopsis FT, is the principle mediator responsible for $\mathrm{NB}$ flowering retardation [11]. In soybean, down-regulation of FT2 $a$ and FT5 $a$ is the major cause for $\mathrm{NB}$ responses [12]. In contrast, multiple NBs accelerate heading in wheat plants grown under SD by inducing FT1 [13]. Genetic studies indicate that the transcriptional changes of $F T$ in response to $\mathrm{NB}$ are predominantly mediated by phytochromes. NB responses are abolished in the phyB mutants of rice [14], phyA mutants of soybean [12] and the phyB and phyC mutants of wheat [13], indicating functional conservation and divergence of phytochromes across different plant species. Further studies indicate circadian clock genes are also involved in NB responses. The up-regulation of FT1 by NB in wheat is dependent on PHOTOPERIOD1 (PPD1), a homolog of PSEUDO RESPONSE REGULATOR 37 (PRR37) in rice, which is a core component of the circadian clock [13]. In poplar (Populus L.), NB induces FT2 to free the shoot growth cessation by suppressing the circadian clock gene LATE ELONGATED HYPOCOTYL 2 (LHY2) [15]. Thus, NB affects plant flowering by controlling different layers of regulators.

Long-noncoding RNAs (lncRNAs) refer to a class of RNA transcripts longer than 200 nt which lack discernable protein coding potential [16, 17]. Compared with mRNAs, lncRNAs have much lower expression levels and sequence conservation [16, 17]. Most IncRNAs harbor spatio-temporal specificity [16, 17]. lncRNAs could be generated from the intergenic, intronic, or coding regions in the sense and antisense directions of mRNA [16, 17]. IncRNAs control the expression of target genes in cis- or trans-acting way $[18,19]$. IncRNAs exert their functions by serving as sponge for miRNAs, functioning as precursors of miRNAs in the cytoplasm [20], or serving as scaffolds of epigenetic regulators to modulate chromatin status in the nucleus $[17,21]$. A few studies indicate that lncRNAs participate in different biological processes, such as flowering, fertility, phosphate metabolism, leaf patterning, nodule formation and phytohormonerelated development. COLD INDUCED LONG ANTI SENSE INTRAGENIC RNAs (COOLAIR) and COLD ASSISTED INTRONIC NONCODING RNA (COL$D A I R)$, two different classes of lncRNAs transcribed from the site of FLOWERING LOCUS C (FLC) in the antisense direction and from the first intron of $F L C$ in the sense direction, respectively, are involved in flowering regulation by repressing $F L C$ via epigenetic modulation [22-24]. LD-SPECIFIC MALE-FERTILITYASSOCIATED RNA (LDMAR) controls the photoperiod-sensitive male sterility in rice under LD [25]. The rice IncRNA INDUCED BY PHOSPHATE STARVATION 1 (IPS1) functions as a target mimic of miR399 to up-regulate the phosphate metabolism gene PHOSPHATE2 (PHO2) when encountered with phosphate starvation [26]. TWISTED LEAF (TL), an antisense lncRNA of OsMYB60, suppresses OsMYB60 via epigenetic chromatin modification to regulate leaf morphology [27]. In Medicago truncatula, the lncRNA EARLY NODULIN 40 (ENOD40) ENOD40 interacts with RNA-BINDING PROTEIN 1 (MtRBP1) to change its location from nuclear speckles to cytoplasmic granules to promote nodule formation [28]. The lincRNA AUXIN REGULATED PROMOTER LOOP (APOLO) regulates the promotor chromatin loop of PINOID (PID) which regulates polar auxin transport [29]. Recently, genome-wide identification of lncRNAs has been performed in a few plants. Huang et al identified 14 lncRNAs co-expressed with 10 pollenassociated coding genes during pollen development in Brassica rapa (B. rapa) [30]. One hundred forty-two lncRNAs may participate in fruit ripening and the 
climacteric in Cucumis melo by regulating the targets involved in auxin signal transduction, ethylene, sucrose biosynthesis and signaling and the ABA signaling pathway [31]. One hundred eighty-five salt stressrelated IncRNAs were obtained in duckweed (Spirodela polyrhiza) [32]. By co-expression analysis, the important lncRNA-mRNA pairs associated with contrasting drought stress responses in two rapeseed cultivators (B. napus) were obtained [33]. These studies have shed lights on the roles of lncRNAs. However, the roles of lncRNAs in flowering regulation remain largely unknown, especially in quinoa (Chenopodium quinoa), an important SD coarse cereal.

Quinoa has been recommended by FAO as "super food" because of the nutritious elements in the seeds [34]. As quinoa is a facultative SD plant $[35,36]$, many photoperiod-sensitive quinoa accessions may encounter with yield penalties when grown across different latitudes. However, for the time being the lncRNAs and corresponding regulatory mechanisms involved in quinoa photoperiodic flowering remain to be elucidated. To this end, in the present study, we investigated the effect of NB on quinoa flowering, and by performing strand-specific RNA sequencing (SS RNA-seq) of the plants grown under NB and SD, we identified the specific coding genes and lncRNAs that may be involved in flowering regulation. Hence, this study provides novel insights into the quinoa flowering molecular mechanisms.

\section{Results}

Effects of short-day and night-break on quinoa flowering time

To evaluate the effects of short-day (SD) and night-break (NB) on quinoa flowering, two quinoa cultivars ("HL1" and "HL2") with different maturation periods were used. Seeds were sown in pots and grown under natural day conditions before photoperiodic treatment. Then, $14 \mathrm{~d}$ old seedlings were transferred into growth chambers and subjected to SD and NB treatment, respectively. After 14 $\mathrm{d}$ photoperiodic entertainment in growth chambers $(28 \mathrm{~d}$ after-sowing, 28 DAS), the floral buds initiated in "HL1" and "HL2" under SD, whereas no visible floral buds found in both cultivars under NB. On 40 DAS, the floral buds size was obviously bigger under SD (Fig. 1a, c) than that under NB (Fig. 1b, d). Thus, we speculated that SD accelerates whereas NB represses floral buds development.

\section{Transcriptome sequencing and identification of IncRNAs in quinoa}

To uncover the roles of lncRNAs in NB responses of quinoa, seedlings of "HL1" were grown in growth chamber and treated by SD and NB as described in section Methods. The leaf samples of "HL1" were collected on 14 DAS (SD1), 26 DAS (SD2) under SD, and 28 DAS under NB. Sampling was performed with three biological replicates for each time point. Then the samples were subjected to total RNA extraction and transcriptome analysis. Through SS RNA-seq on an

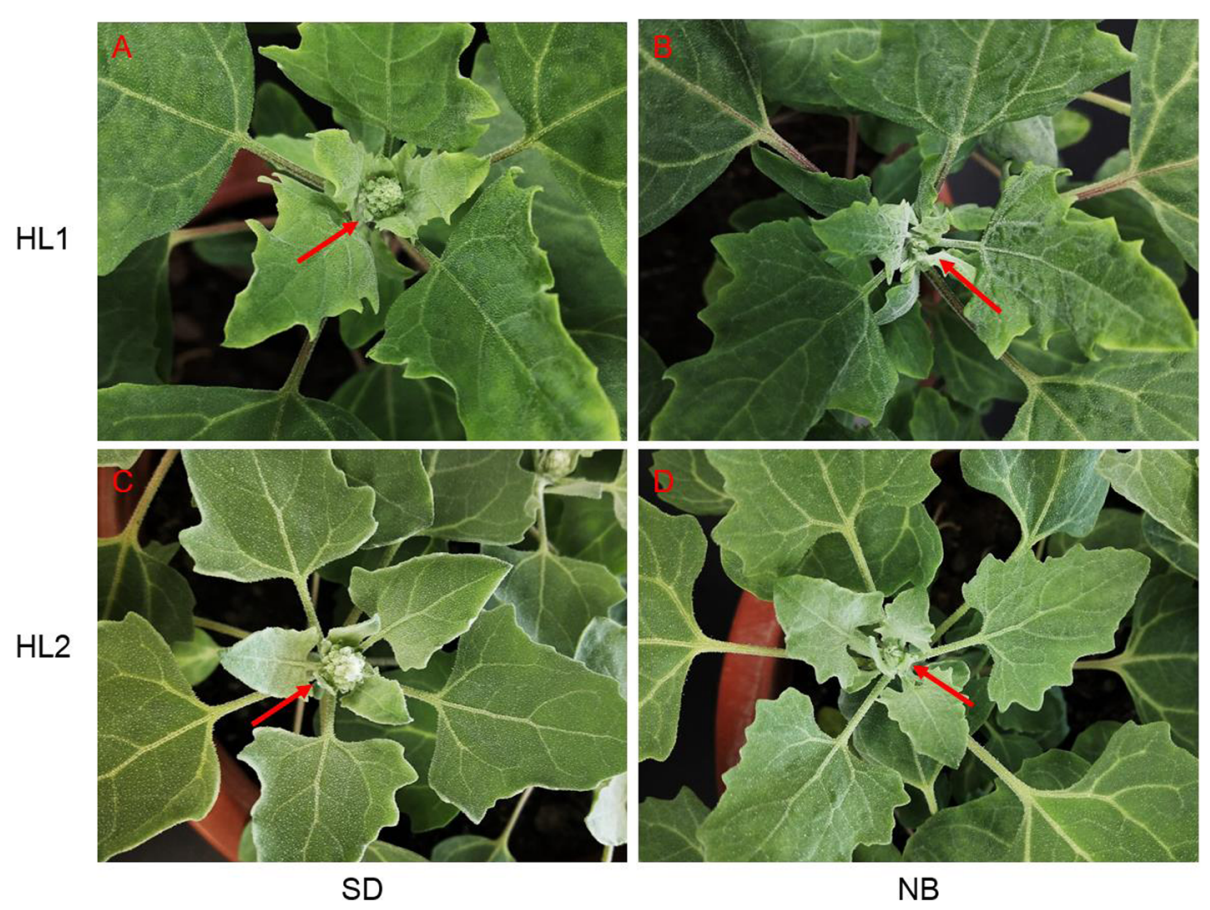

Fig. 1 Phenotypes of the floral buds in two quinoa cultivars under SD and NB. On 40 DAS, the floral buds were apparently visible in cultivars "HL1" (a) and "HL2" (c) under SD, whereas under NB, the floral buds were very tiny in cultivars "HL1" (b) and "HL2" (d) 
Table 1 Summary of the clean reads obtained from 9 strand-specific cDNA libraries

\begin{tabular}{llllllll}
\hline Sample & Clean reads & Clean bases & GC Content & Q30 & Mapped Reads & Unique Mapped Reads & Multiple Map Reads \\
\hline SD1-1 & $120,575,592$ & $18,024,639,138$ & $42.06 \%$ & $93.79 \%$ & $105,438,405(87.45 \%)$ & $77,322,246(64.13 \%)$ & $28,116,159(23.32 \%)$ \\
SD1-2 & $118,844,120$ & $17,742,199,126$ & $42.33 \%$ & $94.07 \%$ & $103,599,037(87.17 \%)$ & $75,650,640(63.66 \%)$ & $27,948,397(23.52 \%)$ \\
SD1-3 & $109,416,192$ & $16,342,140,838$ & $42.23 \%$ & $93.34 \%$ & $94,815,186(86.66 \%)$ & $69,895,804(63.88 \%)$ & $24,919,382(22.77 \%)$ \\
SD2-1 & $118,152,040$ & $17,646,198,882$ & $42.35 \%$ & $93.17 \%$ & $104,853,815(88.74 \%)$ & $72,776,293(61.60 \%)$ & $32,077,522(27.15 \%)$ \\
SD2-2 & $156,229,454$ & $23,239,577,248$ & $42.31 \%$ & $93.38 \%$ & $138,496,418(88.65 \%)$ & $96,469,015(61.75 \%)$ & $42,027,403(26.90 \%)$ \\
SD2-3 & $107,354,748$ & $16,024,541,498$ & $42.52 \%$ & $90.79 \%$ & $91,987,548(85.69 \%)$ & $64,905,652(60.46 \%)$ & $27,081,896(25.23 \%)$ \\
NB-1 & $107,521,308$ & $16,067,918,002$ & $42.84 \%$ & $91.25 \%$ & $91,911,056(85.48 \%)$ & $67,788,130(63.05 \%)$ & $24,122,926(22.44 \%)$ \\
NB-2 & $119,435,422$ & $17,792,985,836$ & $42.53 \%$ & $93.07 \%$ & $104,464,425(87.47 \%)$ & $76,143,854(63.75 \%)$ & $28,320,571(23.71 \%)$ \\
NB-3 & $117,442,040$ & $17,505,052,862$ & $42.31 \%$ & $94.01 \%$ & $103,617,559(88.23 \%)$ & $75,619,123(64.39 \%)$ & $27,998,436(23.84 \%)$ \\
\hline
\end{tabular}

Illumina Hiseq4000 platform, an average of more than 16.02 GB data of 9 cDNA libraries were obtained (Table 1). The Q30 value was more than $90.79 \%$ for each library (Table 1 ). Subsequently, the clean reads were mapped to the quinoa reference genome [37] using HISAT2 software [38], and the mapping rate was higher than $85.48 \%$ for each library (Table 1). Based on the mapping results, StringTie [39] was used to re-construct the transcripts and predict the novel genes. The

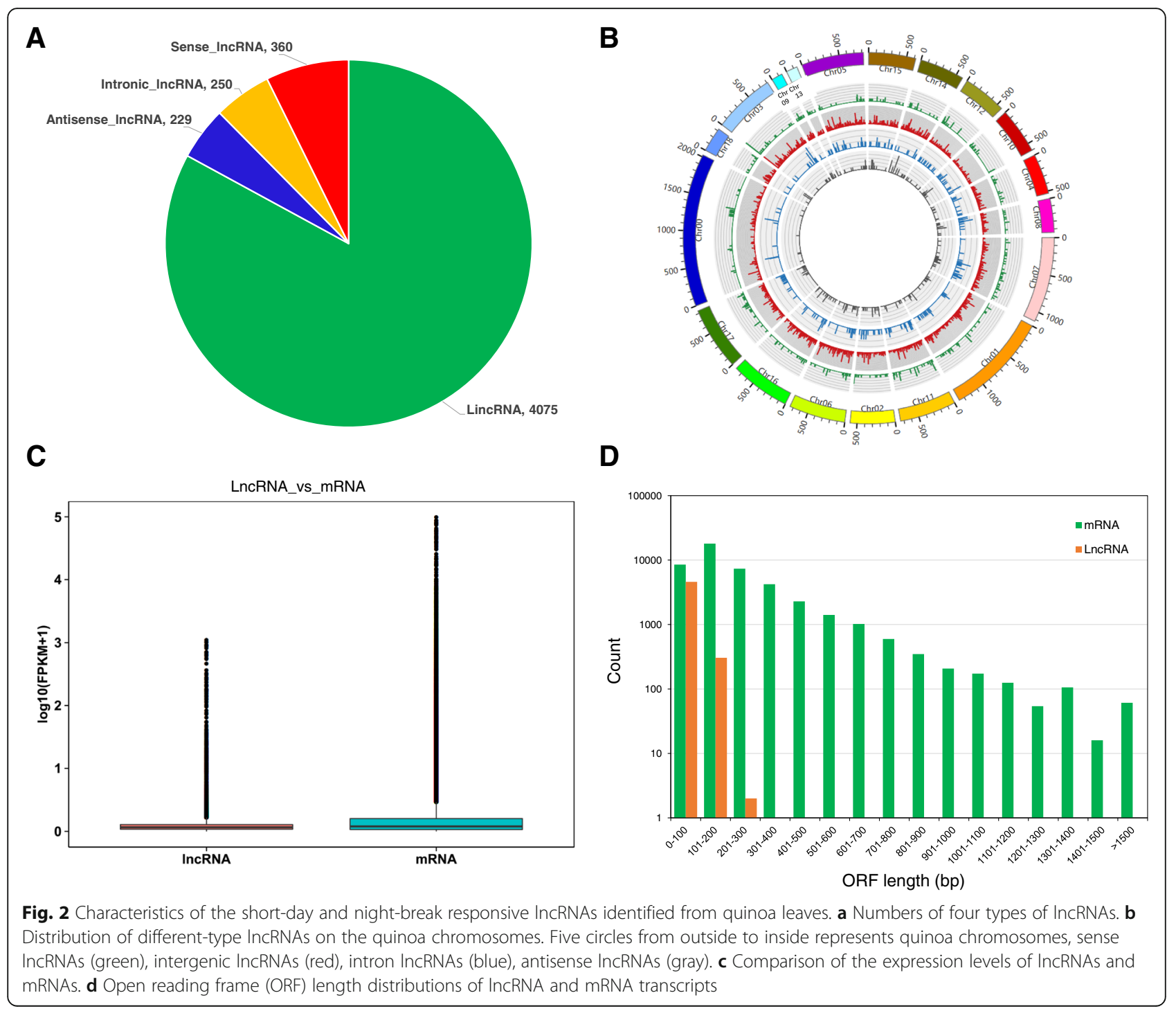


assembled transcripts were annotated by Gffcompare program [40], and the unannotated transcripts were further used for long-noncoding RNA (lncRNA) prediction. Based on the criteria described in section Methods, a total of 4914 lncRNAs were identified. According to their genomic origins, 4075 intergenic lncRNAs (lincRNAs), 250 intronic lncRNA, 360 sense lncRNAs, and 229 antisense lncRNAs were identified (Fig. 2a), respectively. By performing BLAST on lncRNAs database, we found only 605 lncRNAs were annotated in the NONCODE database [41], and most (87\%) of the quinoa lncRNAs were novel genes. Meanwhile, as shown in Fig. 2b, various types of lncRNAs were evenly distributed over the whole quinoa genome at the density of 3.5 per $\mathrm{Mb}$.

The relative gene expression level of each mRNA and lncRNA was normalized to Fragments Per Kilobase of transcript sequence per Millions base pairs sequenced (FPKM) value [42]. Based on the gene expression profiles, Spearman correlation analysis suggested that, three biological replicates were highly related with correlation efficiencies larger than $0.94\left(R^{2}>0.94\right)$, and were readily clustered into the same clade (Additional file 1: Fig. S1). Besides, SD1 and NB samples were clustered as neighboring clades, distant from SD2 clade (Fig. S1), indicating some transcriptional changes from SD1 to SD2 were recovered by NB. Statistics of mRNA and lncRNA expression profiles showed that lncRNAs were expressed at much lower levels compared with mRNAs (Fig. 2c). The open reading frame (ORF) lengths comparison results showed that, IncRNAs predominately harbored $100 \mathrm{bp}$-long ORFs, significantly shorter than that of
mRNAs (Fig. 2d). Based on a criterion of average FPKM of three replicates $>0.1$, we found 1698 lncRNAs were commonly expressed over different time points, and 361, 569 and 415 lncRNAs were specifically expressed at SD1, SD2, and NB, respectively (Fig. 3a). Meanwhile, based on the threshold of average FPKM of three replicates $>1,14,056$ mRNAs were detected across the whole time points (Fig. 3b). More genes (762) were specifically expressed at SD2 compared with the expressed genes at SD1 (173) and NB (605) (Fig. 3b). Coincidently, more lncRNAs and mRNAs were specifically expressed at SD2 than at NB, indicating SD may promote the expression of flowering genes but NB may repress this effect.

\section{Differential expression analysis of IncRNAs and mRNAs}

DESeq2 [43] was employed to explore the differentially expressed lncRNAs (DE lncRNAs) and mRNAs (DEGs) between different groups with thresholds of FDR $<0.05$ and $\mid \log 2$ (fold change) $\mid \geq 1$. In total, $91 \mathrm{DE}$ lncRNAs and 1401 DEGs were obtained in comparisons of SD1_vs_ SD2 and SD2_vs_NB (Table 2, Additional file 2: Table S1, Additional file 3: Table S2). Sixty-four lncRNAs were differentially expressed in SD1_vs_SD2, out of which 40 and 24 lncRNAs were up- and down-regulated in SD1_ vs_SD2 (Table 2), respectively. After transferring from SD2 to NB, the expression levels of 20 and 31 lncRNAs were increased and decreased, respectively (Table 2). DEGs analysis showed that 693 and 309 genes were upand down-regulated in SD1_vs_SD2, and 315 and 379 were up- and down-regulated in SD2_vs_NB,
A

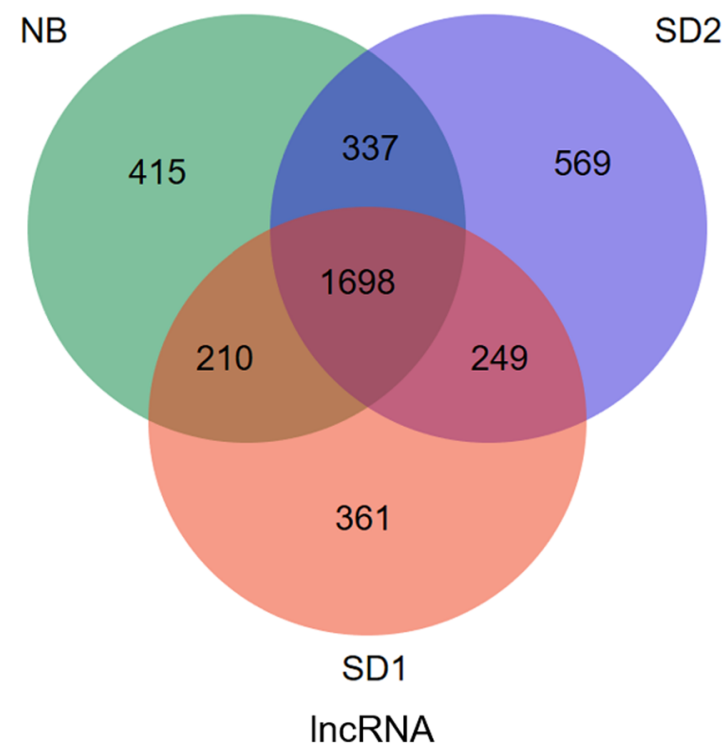

B

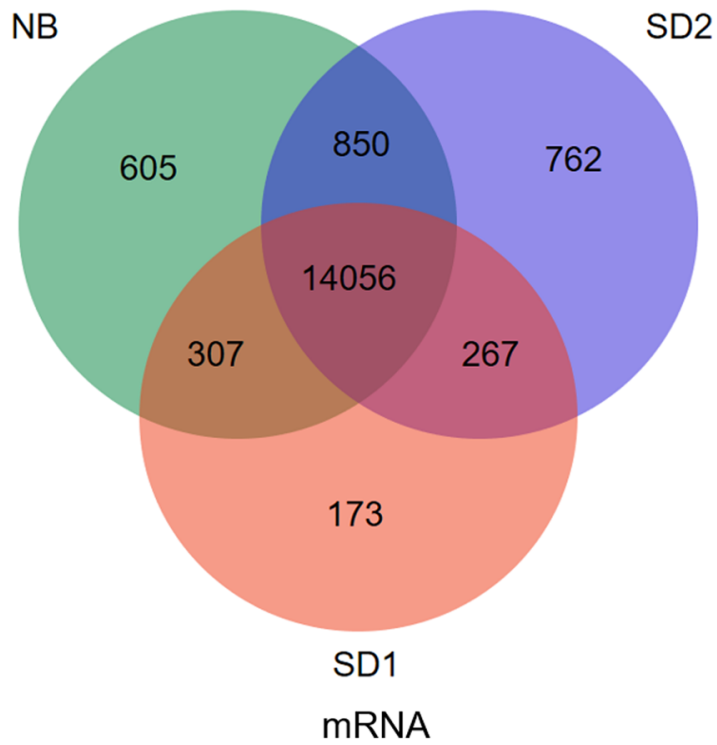

Fig. 3 Venn diagrams showing the numbers of commonly and specifically expressed IncRNAs and mRNAs in different groups. a Numbers of commonly and specifically expressed IncRNAs in SD1, SD2 and NB based on a cutoff of FPKM>0.1. b Numbers of commonly and specifically expressed mRNAs in SD1, SD2 and NB based on a cutoff of FPKM>1. The FPKM value was denoted as the average value of three replicates 
Table 2 Brief review of the differentially expressed IncRNAs and mRNA between different groups

\begin{tabular}{|c|c|c|c|c|c|c|}
\hline \multirow{2}{*}{ Comparison } & \multicolumn{3}{|c|}{ DE IncRNAs } & \multicolumn{3}{|l|}{ DEGs } \\
\hline & total & up & down & total & up & down \\
\hline SD1_Vs_SD2 & 64 & 40 & 24 & 1002 & 693 & 309 \\
\hline SD2_vs_NB & 51 & 20 & 31 & 694 & 315 & 379 \\
\hline
\end{tabular}

respectively (Table 2). These results indicated that many lncRNA and mRNA genes tended to be activated by SD whereas repressed by NB.

\section{Functional annotation of the differentially expressed IncRNAs in response to SD and NB}

To investigate the potential roles of DE lncRNAs, we performed functional annotation of the DE lncRNAs targets. The cis targets were selected from the mRNAs located at less than $100 \mathrm{kbp}$ downstream and upstream of each IncRNA. The trans targets were determined with cutoffs of Pearson's correlation coefficient $>0.9$ or $<-0.9$ and $P$ value $<0.01$ between the expression profiles of mRNAs and lncRNAs. Co-expression and co-location analysis demonstrated that 5917 targets were regulated by the up-regulated lncRNAs in SD1_vs_SD2, including 29,401 IncRNA-mRNA pairs (Additional file 4: Table S3). Kyoto Encyclopedia of Genes and Genomes (KEGG) enrichment analysis indicated that these targets were abundant in pathways of "Ribosome biogenesis in eukaryotes" and "Photosynthesis-antenna proteins" (Fig. 4a). 5100 targets and 9340 lncRNA-mRNA pairs were predicted to be related with the down-regulated lncRNAs in SD1_vs_SD2 (Additional file 5: Table S4). "Photosynthesis-antenna proteins" was the only significantly enriched KEGG pathway for these target genes (Fig. 4b). In the network of the up-regulated lncRNAs in SD2_vs NB, 4736 targets and 8420 lncRNA-mRNA pairs were contained (Additional file 6: Table S5). KEGG pathways including "Photosynthesis-antenna proteins" and

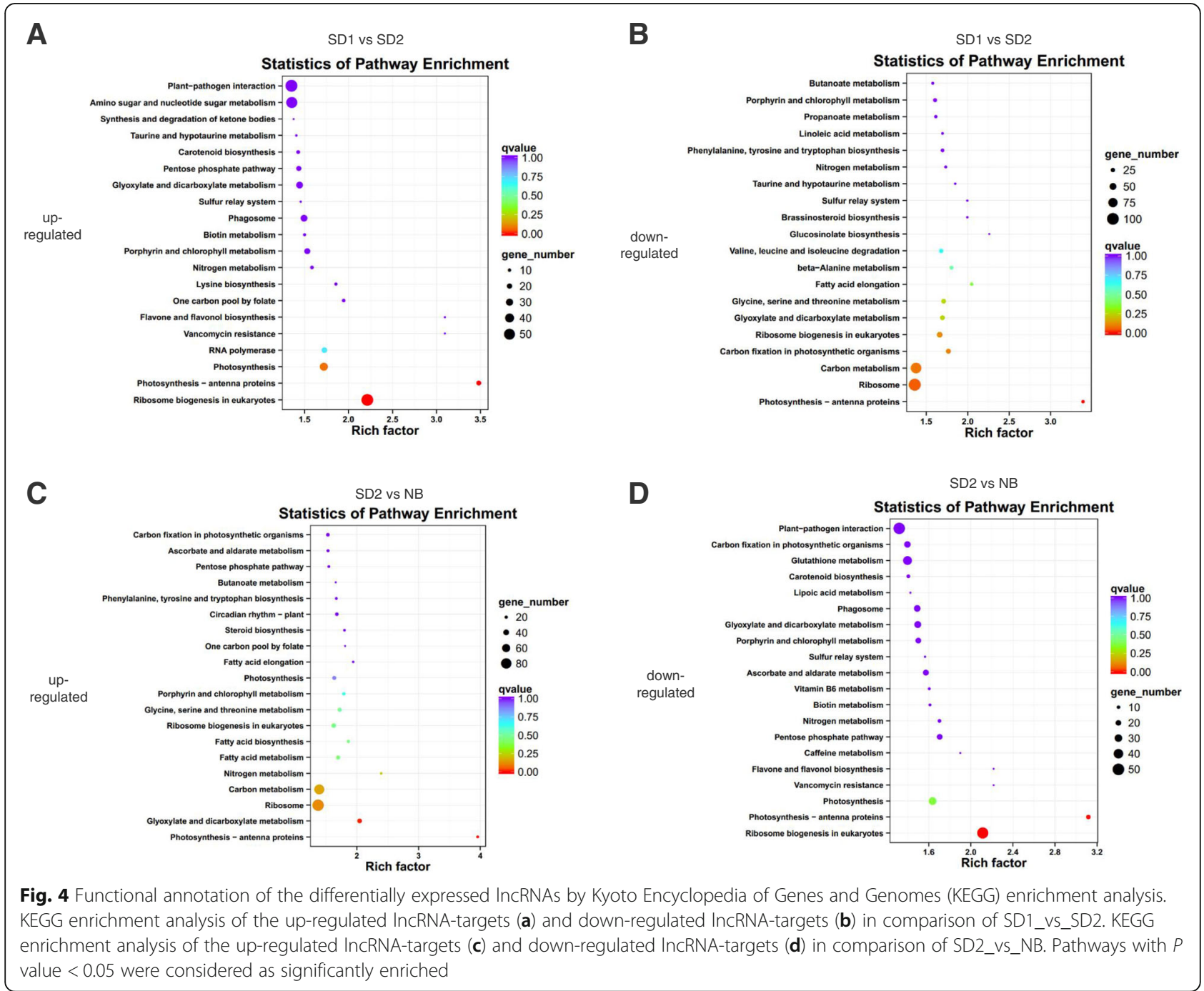


"Glyoxylate and dicarboxylate metabolism" were highly related with these targets (Fig. 4c). 5929 targets and 20, 438 IncRNA-mRNA pairs were involved in the network of the down-regulated lncRNAs in SD2_vs_NB (Additional file 7: Table S6). "Ribosome biogenesis in eukaryotes" and "Photosynthesis-antenna proteins" were the most significantly enriched KEGG pathways for these targets (Fig. 4d).

In addition, to further reveal the roles of DE IncRNAs, we performed Gene Ontology (GO) enrichment analysis to obtain the top 20 significantly enriched GO terms of biological processes, cellular components and molecular functions. With regard to the targets of up-regulated lncRNAs under SD, the most significantly enriched GO terms for biological processes were "pentose-phosphate shunt", "isopentenyl diphosphate biosynthetic process, methylerythritol 4-phosphate pathway", photosynthesis, light harvesting", "nucleosome assembly", "glucose catabolic process", "rRNA processing", "photosynthesis", "cysteine biosynthetic process" and "tetrahydrofolate interconversion" (Fig. 5a). The GO terms including "chloroplast stroma", "photosystem II oxygen evolving

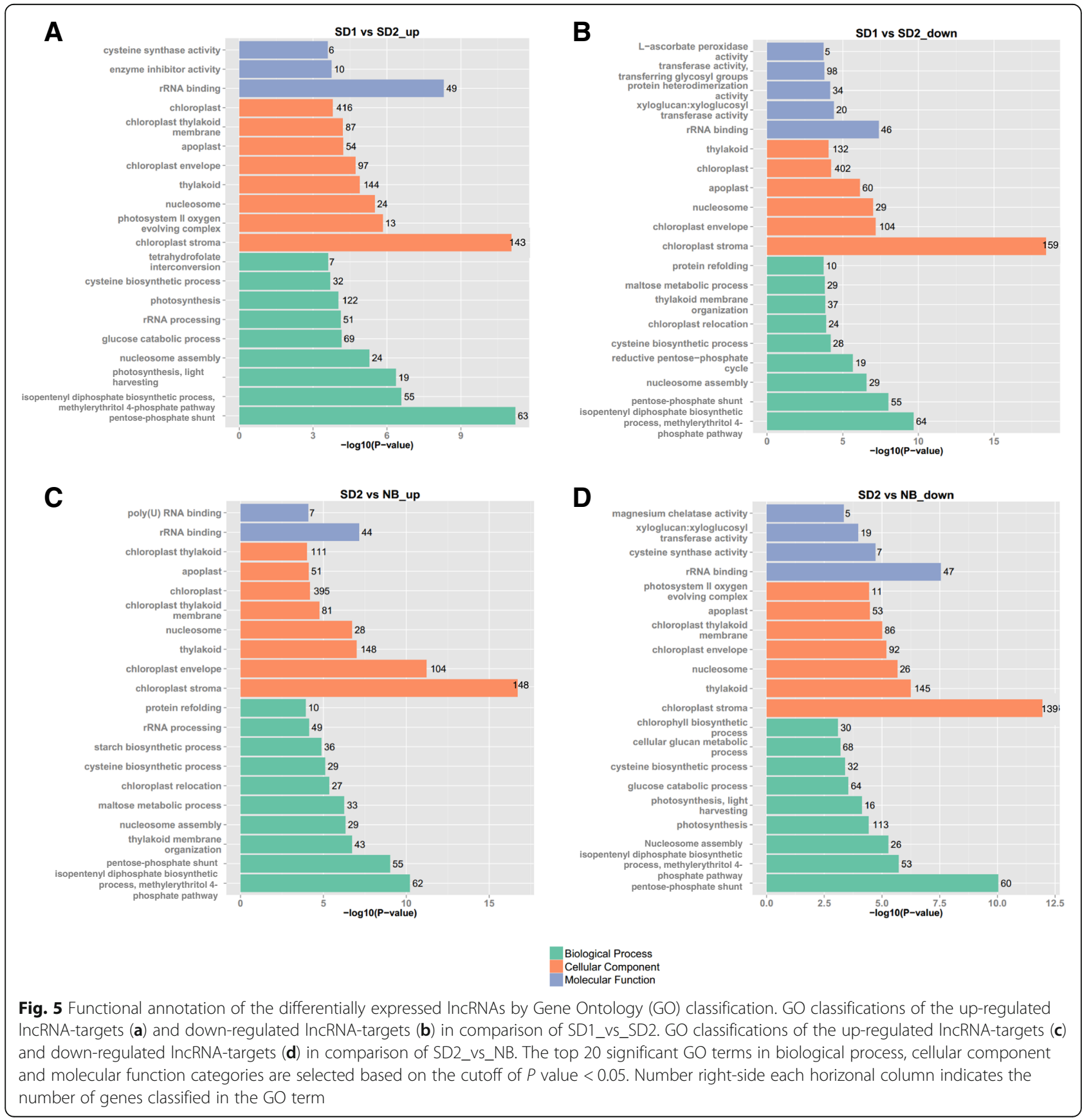


complex", "nucleosome", "thylakoid", "chloroplast envelope", "apoplast", "chloroplast thylakoid", "membrane" and "chloroplast" were enriched in cellular component category (Fig. 5a). The GO terms of "rRNA binding", "enzyme inhibitor activity" and "cysteine synthase activity" were highly represented in molecular functions (Fig. 5a). The targets of the down-regulated lncRNAs under SD were abundant in 9, 6 and 5 GO terms of biological process, cellular component, and molecular function categories, respectively. As far as biological processes were concerned, "isopentenyl diphosphate biosynthetic process, methylerythritol 4-phosphate pathway", "pentose-phosphate shunt", "nucleosome assembly", "reductive pentose-phosphate cycle", "cysteine biosynthetic process", "chloroplast relocation", "thylakoid membrane organization", "maltose metabolic process", and "protein refolding" were the most important GO terms (Fig. 5b). The 6 GO terms such as "chloroplast stroma", "chloroplast envelope", "nucleosome", "apoplast", "chloroplast" and "thylakoid" were highlighted in cellular components (Fig. 5b). The GO terms like "rRNA binding", "xyloglucan: xyloglucosyl transferase activity", "protein heterodimerization activity", "transferase activity, transferring glycosyl groups" and "L-ascorbate peroxidase activity" were predominant in molecular function category (Fig. 5b).

GO enrichment analysis of the targets of upregulated lncRNAs under NB showed that "isopentenyl diphosphate biosynthetic process, methylerythritol 4-phosphate pathway", "pentose-phosphate shunt", "thylakoid membrane organization", "nucleosome assembly", "maltose metabolic process", "chloroplast relocation", "cysteine biosynthetic process", "starch biosynthetic process", "rRNA processing" and "protein refolding" were highly represented in biological processes (Fig. 5c). GO terms "chloroplast stroma", "chloroplast envelope", "thylakoid", "nucleosome", "chloroplast thylakoid membrane", "chloroplast", "apoplast" and "chloroplast thylakoid" showed high representativeness of cellular components (Fig. 5c). With regard to molecular function category, targets were involved in "rRNA binding" and "poly (U) RNA binding" (Fig. 5c). The targets of down-regulated lncRNAs under NB were significantly enriched in 9, 7 and 4 GO terms of biological process, cellular component, and molecular function categories, respectively. The biological process GO terms contained "pentose-phosphate shunt", "isopentenyl diphosphate biosynthetic

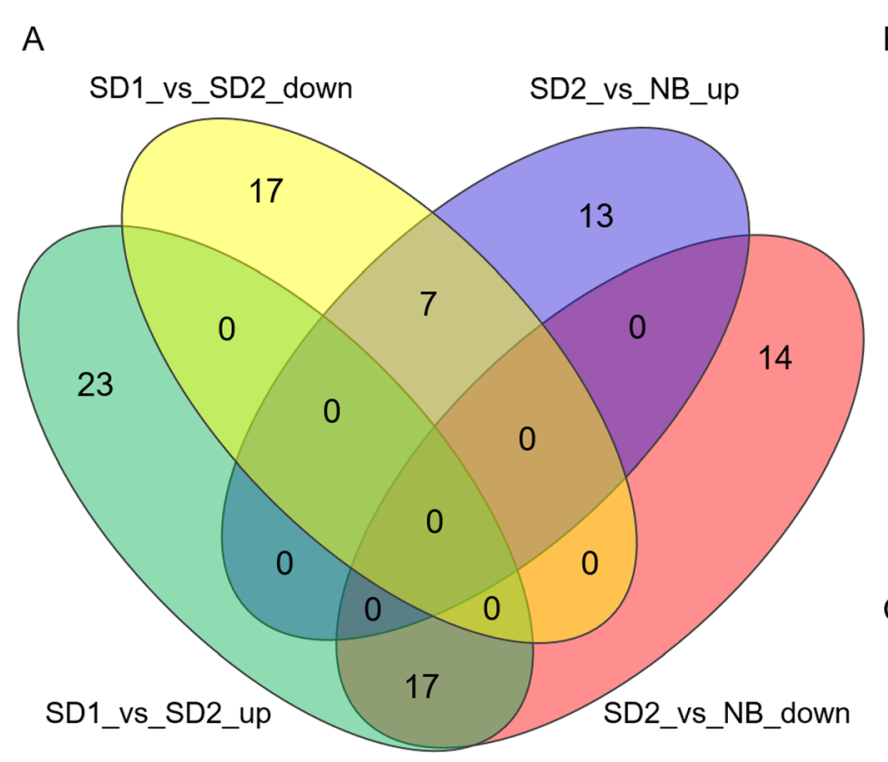

B

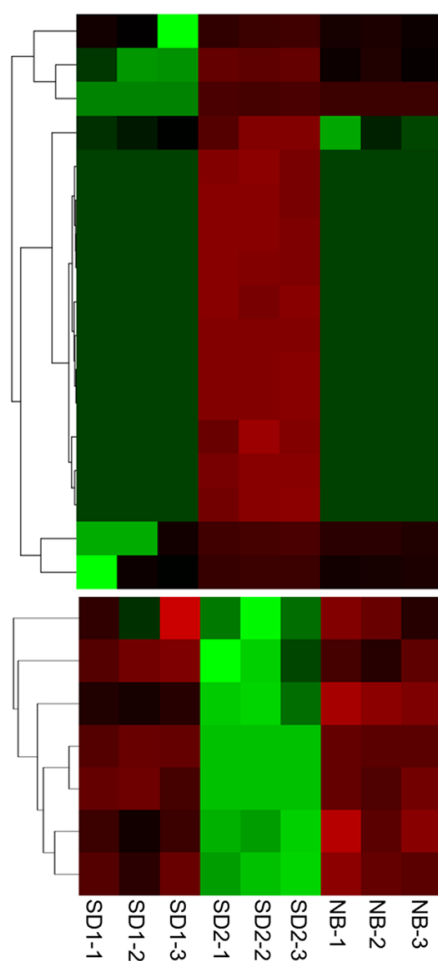

MSTRG.8116.1 MSTRG.39390.2 MSTRG.69967.1 MSTRG.10889.3 MSTRG.10578.1 MSTRG.28884.1 MSTRG.30736.1 MSTRG.47393.1 -2 MSTRG.3107.2 MSTRG.69967.2 MSTRG.24870.1 MSTRG.53484.1 MSTRG.59128.3 MSTRG.14725.1 MSTRG.48364.2 MSTRG.27734.1 MSTRG.29153.1 MSTRG.5158.2 MSTRG.3121.1 MSTRG.29609.4 MSTRG.32307.3 MSTRG.53750.2 MSTRG.11610.1 MSTRG.29609.1 
process, methylerythritol 4-phosphate pathway", "nucleosome assembly", "photosynthesis", "photosynthesis, light harvesting", "glucose catabolic process", "cysteine biosynthetic process", "cellular glucan metabolic process" and "chlorophyll biosynthetic process" (Fig. $5 \mathrm{~d})$. With regard to cellular components, "chloroplast stroma", "thylakoid", "nucleosome", "chloroplast envelope", "chloroplast thylakoid membrane", "apoplast" and "photosystem II oxygen evolving complex" were highly represented (Fig. 5d). As far as molecular functions were concerned, "rRNA binding", "cysteine synthase activity", "xyloglucan: xyloglucosyl transferase activity" and "magnesium chelatase activity" were significantly enriched (Fig. 5d).
Prediction of the putative positive and negative flowering regulatory IncRNAs

According to the flowering time illustrated above, SD and NB exerted positive and negative effects on quinoa flowering, respectively. To ascertain which lncRNAs were probably involved in quinoa flowering, we analyzed the transcriptional profiles of the $91 \mathrm{DE}$ lncRNAs. Through venn diagram analysis, we found that most of the $\mathrm{DE}$ lncRNAs (67 out of 91) were solely affected by SD or NB, and no DE IncRNAs were constantly induced or repressed by both SD and NB (Fig. 6a). Interestingly, we noticed that the expression patterns of 24 DE lncRNAs displayed opposite trends under SD compared with NB (Fig. 6a). Based on the expression profiles, we inferred that the $17 \mathrm{SD}$-induced

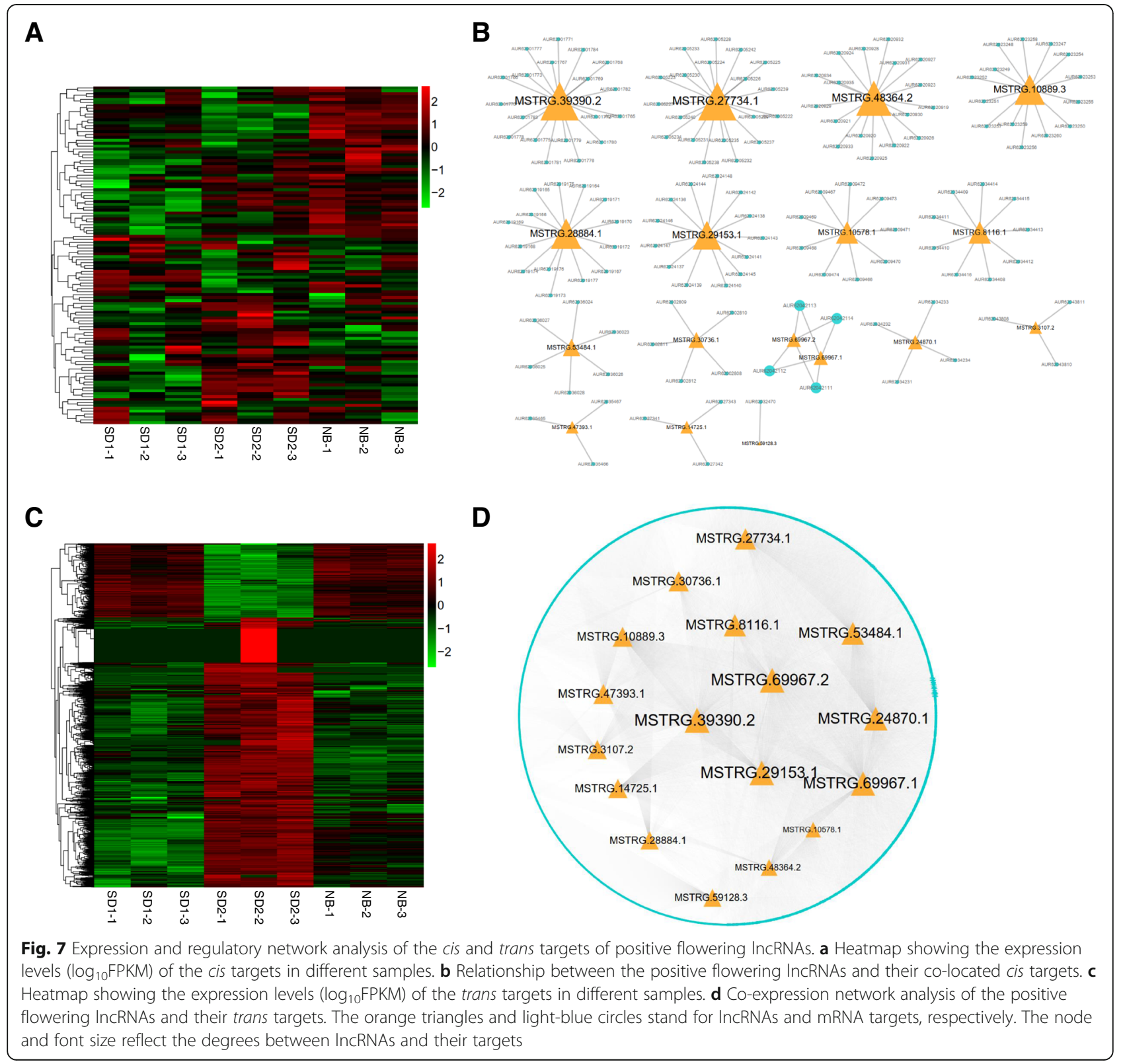


but NB-repressed lncRNAs were the putative positive flowering regulator (Fig. 6b). On the other hand, the 7 lncRNAs that repressed by SD but activated by NB were predicted to be the putative flowering repressors (Fig. 6c).

Further, to understand the functions of the lncRNAs, we investigated the regulatory networks of the putative flowering lncRNAs. With regard to the 17 positive flowering lncRNAs, 143 cis targets with 147 cis-acting lncRNA-mRNA pairs and 4034 trans targets with 16,844 trans-acting lncRNA-mRNA pairs were selected, respectively (Fig. 7) (Additional file 8: Table S7). Cis-acting network analysis indicated that the largest node MSTRG.39390.2 co-located with 19 targets and MSTR G.59128.3 only co-located with 1 target (Fig. 7a-b). Notably, a few important plant growth and development related transcription factors (TFs) were identified among the cis targets. For example, 3 SCARECROW-like genes of GRAS family (AUR62001765, AUR62001766, AUR62001768) co-located with MSTRG.39390.2; 1 WOX (WUSCHEL-related homeobox) (AUR62035466) colocated with MSTRG.47393.1. According to the transacting network of the positive flowering lncRNAs, the largest node MSTRG.39390.2 and smallest node MSTR G.10578.1 were predicted to regulate 1495 and 343 trans targets, respectively (Fig. 7c-d). Moreover, we found that 1 mRNA could be the common target of 1 to 14 lncRNAs and 423 mRNA genes were correlated with more than 10 lncRNAs (Fig. 7d). The expression heatmap demonstrated that most of these trans targets harbored the same expression trend with the lncRNAs (Fig. 7c). Functional annotation by KEGG enrichment analysis suggested that no pathways were significantly enriched for cis targets, whereas the trans target genes were highly abundant in pathways "Ribosome biogenesis in eukaryotes", "Photosynthesis-antenna proteins" and "Photosynthesis" (Table 3).

For the 7 putative flowering repressor lncRNAs, 50 cis targets with 57 cis-acting lncRNA-mRNA pairs and 2615 trans targets with 3985 trans-acting lncRNA-mRNA pairs may be correlated with these lncRNAs (Fig. 8)
(Additional file 9: Table S8). From the cis-acting network we found that the largest node MSTRG.5158.2 colocated with 18 targets, while the smallest nodes MSTR G.53750.2 and MSTRG.3121.1 only co-located with 1 target (Fig. 8a-b). With regard to the trans co-expression network, the largest node MSTRG.29609.1 and the smallest node MSTRG.5158.2 may interact with 1164 and 44 targets, respectively, and 1 mRNA could be correlated with 1 to 4 lncRNAs (Fig. 8c-d). The expression heatmap showed that about $50 \%$ of these trans targets harbored similar expression trend with the negative flowering lncRNAs (Fig. 8c). KEGG enrichment analysis indicated the cis targets were abundant in pathway "Glutathione metabolism", and the trans targets were significantly enriched in pathways "Photosynthesis-antenna proteins", "Ribosome biogenesis in eukaryotes", "Porphyrin and chlorophyll metabolism" and "Glyoxylate and dicarboxylate metabolism" (Table 3).

\section{Co-expressed network associated with quinoa flowering} Furthermore, by comparing the seed nodes in the positive and negative lncRNAs flowering networks, we noticed that 1653 mRNAs were simultaneously regulated by both types of regulatory lncRNAs, accounting for $39.66 \%(1653 / 4168)$ and $62.12 \%(1653 / 2661)$ of the positive and negative network targets, respectively (Fig. 9a, b). The expression heatmap indicated that most of these targets harbor higher expression levels under SD than under NB (Fig. 9c). Co-expression network analysis demonstrated that the largest node MSTR G.69967.2 was linked with 1176 targets while MSTR G.59128.3 was only co-expressed with 1 target, and the top-5-degree target nodes (Chenopodium_quinoa_newGene 17423, AUR62040917, AUR62018533, AUR62015897, AUR62000858) were regulated by as many as 17 lncRNAs (Fig. 9b). Intriguingly, among the 1653 targets, we found that a few vital plant growth and development related modulators co-expressed with 15 positive and 5 negative putative flowering lncRNAs (Fig. 10a-b). For example, the FLOWERING LOCUS T-

Table 3 KEGG enrichment analysis of the cis and trans targets of the putative positive and negative flowering IncRNAs

\begin{tabular}{|c|c|c|c|c|}
\hline & Targets & KEGG pathway & Pathway ID & Corrected $P$ value \\
\hline \multirow[t]{4}{*}{ Positive flowering IncRNAs } & cis targets & - & - & - \\
\hline & trans targets & Ribosome biogenesis in eukaryotes & ko03008 & $6.19 \mathrm{E}-08$ \\
\hline & & Photosynthesis - antenna proteins & ko00196 & $6.43 \mathrm{E}-05$ \\
\hline & & Photosynthesis & ko00195 & 0.040877 \\
\hline \multirow[t]{5}{*}{ Negative flowering IncRNAs } & cis targets & Glutathione metabolism & ko00480 & 0.002922 \\
\hline & trans targets & Photosynthesis - antenna proteins & ko00196 & $6.30 \mathrm{E}-08$ \\
\hline & & Ribosome biogenesis in eukaryotes & ko03008 & 0.008546 \\
\hline & & Porphyrin and chlorophyll metabolism & ko00860 & 0.016466 \\
\hline & & Glyoxylate and dicarboxylate metabolism & ko00630 & 0.026896 \\
\hline
\end{tabular}




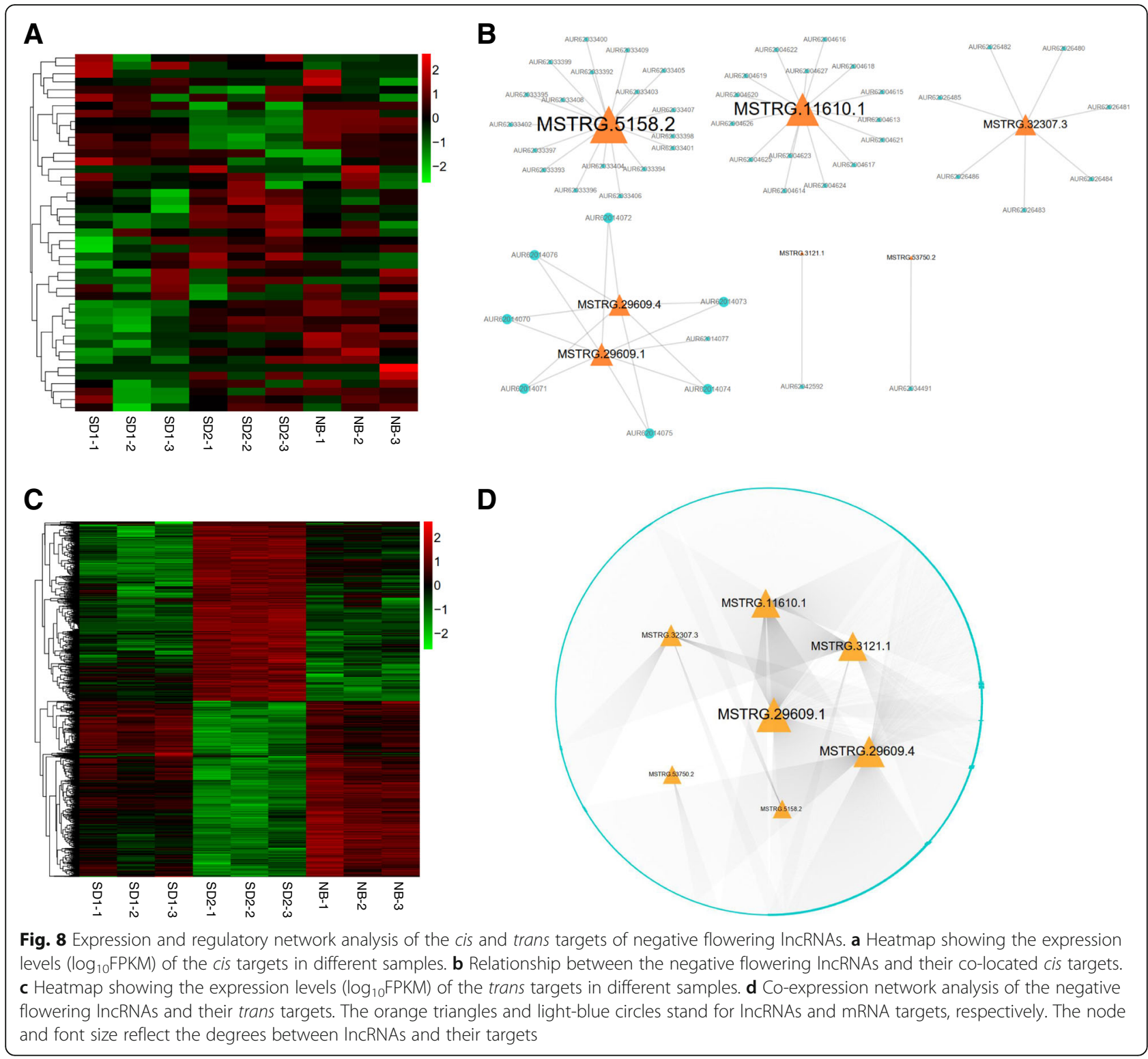

like (FT-like) homolog (AUR62006619), which belongs to the PHOSPHATIDYLETHANOLAMINE-BINDING PROTEIN (PEBP) family and functions as a crucial floral integrator triggering downstream floral organogenesis in plants [10], was predicted to be co-expressed with 13 positive (MSTRG.53484.1, MSTRG.30736.1, MSTR G.39390.2, MSTRG.27734.1, MSTRG.14725.1, MSTR G.47393.1, MSTRG.3107.2, MSTRG.29153.1, MSTR G.24870.1, MSTRG.10889.3, MSTRG.8116.1, MSTR G.69967.1, MSTRG.69967.2) and 1 negative (MSTR G.29609.1) flowering IncRNAs (Fig. 10a-b). Another PEBP member TWIN SISTER of FT-like (TSF-like) homolog (AUR62026433), which shares similar functions with FT, may be regulated by 5 positive (MSTR G.30736.1, MSTRG.39390.2, MSTRG.47393.1, MSTR G.28884.1, MSTRG.69967.2) and 2 negative (MSTR
G.3121.1, MSTRG.29609.1) flowering lncRNAs (Fig. 10ab). AUR62039984, encoding a B-BOX Zinc Finger protein CONSTANS-like (CO-like) which acts upstream of $F T$ in photoperiod-dependent manner [44], was tightly correlated with the flowering activator MSTR G.8116.1 and the flowering repressor MSTRG.32307.3. AUR62009205, an EARLY FLOWERING 3-like homolog which plays important roles in maintaining circadian rhythms and controlling flowering time $[45,46]$, was the trans target of 2 positive (MSTRG.32307.3, MSTR G.3121.1) and 2 negative (MSTRG.39390.2, MSTR G.69967.1) flowering lncRNAs (Fig. 10a-b). The $M Y B-$ type LATE ELONGATED HYPOCOTYL (LHY) is a core component of circadian clock controlling many clock outputs such as flowering and photomorphogenesis [47]. In this study, we found that the quinoa $L H Y$ homolog 


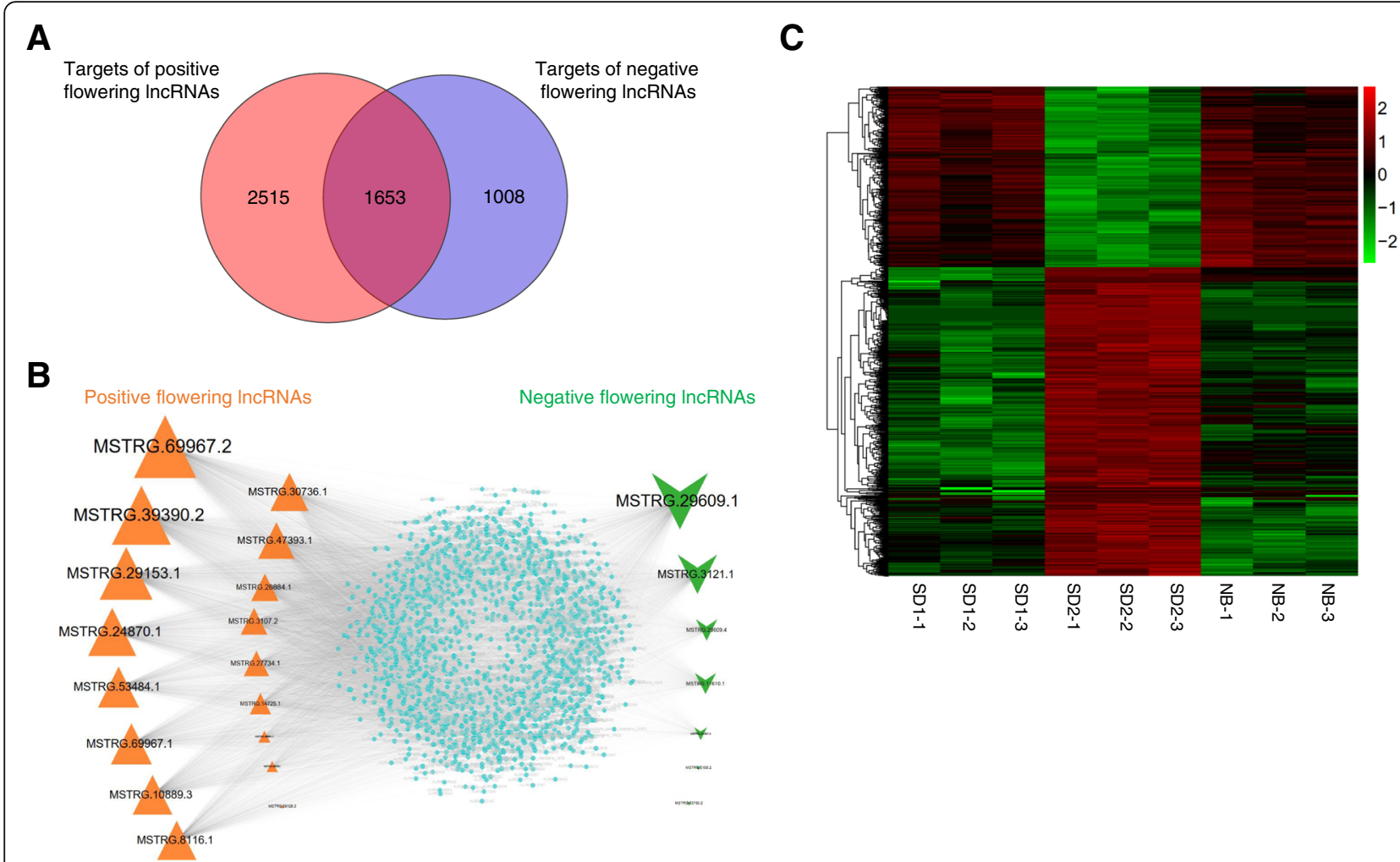

Fig. 9 Identification of the common targets of positive and negative flowering IncRNAs. a Venn diagram showing the number of common and specific targets of positive and negative flowering IncRNAs. $\mathbf{b}$ Co-expression network of 17 positive, 7 negative flowering IncRNAs and their common targets. The orange triangles, green $V$ and light-blue circles represent positive, negative flowering IncRNAs and their common targets, respectively. The node and font size reflect the degrees between IncRNAs and their targets. c Heatmap showing the expression levels $\left(\log _{10}\right.$ FPKM) of the 1653 common targets in different samples

(AUR62004570) was co-expressed with 6 lncRNAs (5 positive lncRNAs: MSTRG.39390.2, MSTRG.27734.1, MSTRG.29153.1， MSTRG.8116.1， MSTRG.69967.1; 1 negative lncRNA: MSTRG.3121.1) (Fig. 10a-b). The bZIP transcription factor ELONGATED HYPOCOTYL 5 (HY5) mediates blue light signaling to modulate circadian clock rhythms and plays multifaceted roles in plant growth and development [48]. The quinoa HY5 homolog AUR62030640 was correlated with 4 positive (MSTR G.39390.2, MSTRG.29153.1, MSTRG.8116.1, MSTR G.69967.2) and 2 negative IncRNAs (MSTRG.32307.3, MSTRG.29609.1) (Fig. 10a-b). Previous studies suggested that the RELATED TO ABI3/VP1 (RAV) family members delay heading time by repressing Heading date $3 a$ $(H d 3 a)$ and GIBBERELLIN 3-OXIDASE1/2 in rice (Oryza sativa) [49]. In the present study, we found that the quinoa RAV1 homolog (AUR62035279) was regulated by 3 lncRNAs ( 2 positive lncRNAs, MSTR G.39390.2, MSTRG.69967.1; 1 negative lncRNA: MSTR G.3121.1) (Fig. 10a-b). Numerous evidences have indicated that photoreceptor PHYTOCHROME A (PHYA) mainly mediates light responses to various ratios of red/ far-red light. In rice, the $p h y A$ mutation in $p h y B$ or $p h y C$ background leads to dramatic early heading [14]. The quinoa PHYA homolog (AUR62003557) was identified as the target of 8 lncRNAs (5 positive lncRNAs, MSTR G.30736.1, MSTRG.29153.1, MSTRG.10889.3, MSTR G.10578.1, MSTRG.69967.2; 3 negative lncRNAs: MSTR G.11610.1, MSTRG.29609.4, MSTRG.29609.1) (Fig. 10ab). In addition, we found another photoreceptor, the blue light photoreceptor CRYPTOCHROME1 homolog (AUR62018922) which is involved in entrainment of circadian clock and photoperiodic flowering [50], was linked with 7 lncRNAs (4 positive lncRNAs, MSTR G.30736.1, MSTRG.47393.1, MSTRG.28884.1, MSTR G.69967.2; 3 negative lncRNAs: MSTRG.11610.1, MSTR G.29609.4, MSTRG.29609.1) (Fig. 10a-b). Consequently, the tight association with these photoreceptors, circadian clock and florigen genes indicates these lncRNAs may play important roles in photoperiodic flowering of quinoa.

\section{Discussion}

Flowering is a vital process closely associated with the final yield of crops. Previous studies have revealed the roles of many protein-coding genes in flowering time 


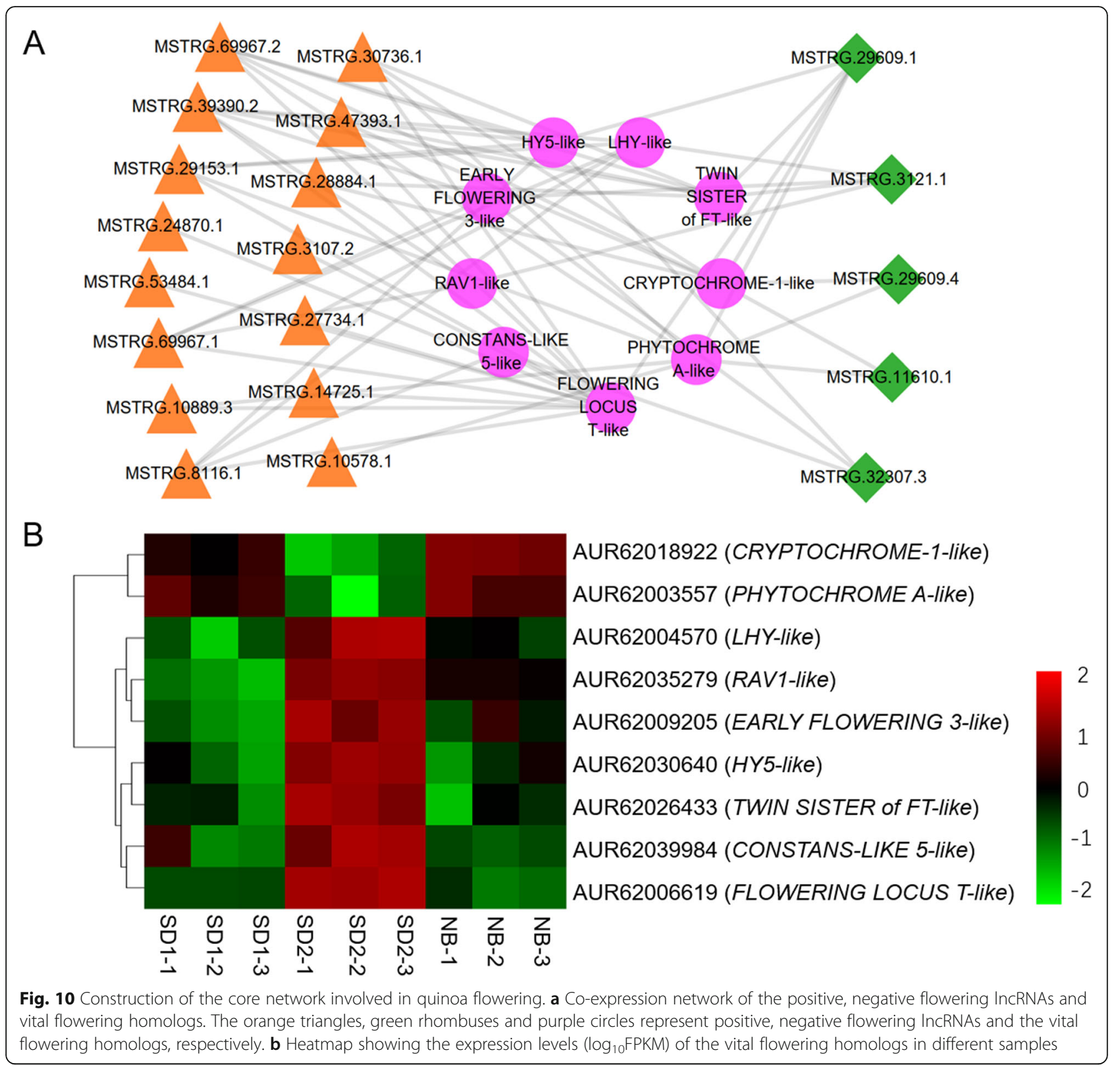

regulation. However, knowledge about the functions of lncRNAs in plant flowering and yield regulation is still limited. Until recently, a few studies have deciphered the pivotal roles of IncRNAs in flowering and yield regulation. In Arabidopsis, IncRNAs COLDAIR and COOLAIR were proven to regulate vernalization and flowering time via epigenic-repression of $F L C$ [23, 24]. In rice, EARLY FLOWERING-COMPLETELY DOMINANT (EF-CD) encodes an antisense lncRNA overlapping with the floral activator OsSOC1 to shorten maturation period without concomitant yield penalty [51]. Mining of specific lncRNAs may provide novel strategies for crop yield improvement.
Quinoa is an annual-flowering crop originated from the low latitude Andeans region. The flowering time and yield of some quinoa cultivars tend to vary when introduced into different countries [52]. However, the flowering regulators, especially the lncRNAs mediated flowering networks, remain to be revealed. SD combined with NB approach is an effective way for exploring the floral regulators. In this study, we found NB repressed the quinoa floral buds development in contrast to the flowering-promoting effects of SD (Fig. 1a-d). These results were consistent with the observations in other SDPs such as rice [11] and soybean [12], indicating the conserved flowering-repressing effects of NB on SDPs. 
To uncover the specific lncRNAs involved in NB responses, we compared the transcriptome differences between plants grown under SD and NB. By highthroughput sequencing, we identified 4914 lncRNAs from quinoa leaf tissues, less than the number of lncRNAs identified from tomato (Solanum lycopersicum) [53] but more than that identified from Cucumis melo [31], Cassava (Manihot esculenta) [54] and peanut (Arachis hypogaea) [55]. Meanwhile, only 605 (12.3\%) shared similar sequences with the known lncRNAs in the NONCODE database. We presumed that the lncRNAs number and sequence conservation may be highly associated with tissue specificity and plant species. In this study, we identified 91 DE lncRNAs, most of which $(73.6 \%)$ were affected solely either by SD or NB. Considering the contrary effects of SD and NB, we neglected this part of DE lncRNAs, and speculated that 17 and 7 lncRNAs, affected by both SD and NB in antagonizing manner, were the putative positive and negative flowering regulators, respectively (Fig. 6a-c).

To investigate the roles of the lncRNAs, we performed KEGG functional enrichment analysis of the cis and trans targets of the putative flowering lncRNAs. Pathway "Glutathione metabolism" was specific for the cis targets of negative flowering lncRNAs. "Photosynthesis" was the specific pathway for the trans targets of positive flowering lncRNAs, while "Porphyrin and chlorophyll metabolism" and "Glyoxylate and dicarboxylate metabolism" were specific for the trans targets of negative flowering lncRNAs (Table 3). These specific pathways may provide valuable cues for investigating the roles of these flowering lncRNAs in future.

Comparison of the trans targets of positive and negative flowering lncRNAs revealed that a considerable number of targets (1653 genes) co-expressed with both types of IncRNAs (Fig. 9a), indicative of competing flowering regulatory networks in quinoa. As supporting evidences, annotation of the common targets revealed a few floral homologs which may function as vital mediators of the antagonizing lncRNAs. Flowering time is largely influenced by endogenous circadian clock and external light conditions which are output to the final integrators $F T$ in leaves [4]. As discovered in a lot of plant species, there are multiple $F T$ homologs with highly conserved sequences in their genomes. However, different $F T$ homologs may experience functional diversification because of the varied expression patterns [56]. For example, in rice there are $11 F T$ homologous genes, among which both $H d 3 a$ and RFT1 are floral activators [11, 57]. However, only $H d 3 a$ showed obvious expression suppression by NB [11], which is the principal cause of the NB effect in rice. Ten FT homologs are isolated in soybean genome, yet, according to the expression pattern they are divided into SD-, LDspecific and photoperiod-independent groups [58]. Functional characterization suggests that GmFT1a and GmFT2a/5a have opposite effects in flowering of soybean [58]. In Chenopodium rubrum (C. rubrum), a close relative of quinoa, CrFTL1 is SD-inducible and inhibited by NB, whereas CrFTL2 is constitutively expressed [59]. In allotetraploid quinoa, 11 putative FT homologs have been identified [60]. However, which are NB-responsive is not determined. In this study, we found that only AUR62006619 (FT-like) and AUR62026433 (TSF-like) were significantly affected by NB (Fig. 10). AUR62006619 and AUR62026433 were down-regulated by 8 -fold and 2-fold, respectively, under NB than under SD. Thus, we speculated that these two $F T$ homologs are probably the essential mediators for $\mathrm{NB}$ responses in quinoa. Co-expression network analysis showed that the quinoa FT-like homolog (AUR62006619) and TSF-like homolog (AUR62026433) were regulated by 14 and 7 lncRNAs, respectively. Noteworthy, both of these two quinoa $F T$ homologs were commonly regulated by 5 lncRNAs (4 positive lncRNAs: MSTRG.30736.1, MSTR G.39390.2, MSTRG.47393.1, MSTRG.69967.2; 1 negative lncRNAs: MSTRG.29609.1) (Fig. 10), suggesting these two homologs may function through partially overlapped signal cascade.

The B-BOX type $C O$, a direct activator of $F T$, functions as the output of circadian clock [61]. In rice, the transcriptional level of $C O$ homolog- $H D 1$ is not obviously changed by NB [11]. However, different scenarios are observed in other plants. For instance, in Chrysanthemum (Chrysanthemum morifolium), the expression of $\mathrm{CmCOL1}$ is slightly reduced by NB [62]. In $C$. rubrum, two CrCOLs genes were down-regulated by dark-light transition [59]. Similarly, we found that 1 out 6 CO-like homologs (AUR62039984) in quinoa was down-regulated by NB. These results indicate the specific roles of $\mathrm{CO}$ homologs of different plants in NB responses. Coincidently, three circadian clock related genes, LHY-like (AUR62004570), ELF3-like (AUR62009205) and HY5-like (AUR62030640), were also repressed by NB. In addition, LHY-like, HY5-like and CO-like were commonly regulated by one IncRNA (MSTRG.8116.1) (Fig. 10). These results imply the possible positive roles of these circadian clock genes on quinoa CO-like. Photoreceptors are essential for perceiving light signals to regulate circadian clock and flowering time. Phytochromes and Cryptochromes are responsible for red/far-red and blue light perceiving, respectively. In quinoa, there are 6 Phytochromes (2 PHYA, 2 PHYB and $2 P H Y C$ ) and 4 Cryptochromes (2 CRY1 and $2 C R Y 2$ ) [60]. In this study, we found 1 PHYA and $1 C R Y 1$ were 
specifically up-regulated by NB and were commonly coexpressed with as many as 5 lncRNAs (MSTRG.69967.2, MSTRG.30736.1, MSTRG.29609.1, MSTRG.29609.4, MSTRG.11610.1 and MSTRG.30736.1) (Fig. 10). It is of note that MSTRG.69967.2, MSTRG.30736.1 and MSTR G.29609.1 also participated in regulating the two $F T$ homologs (Fig. 10). Thus, these data suggested PHYA and $C R Y 1$ may regulate flowering time by controlling the two FT homologs. Together, these putative flowering lncRNAs may mediate NB responses by regulating the key flowering genes. We believe further functional investigation on these lncRNAs will shed light on their roles in quinoa flowering.

\section{Conclusion}

In summary, in this study we performed SS RNA-seq to explore genome-wide lncRNAs in quinoa. By analyzing the transcriptome data under SD and NB, we predict the specific lncRNAs that may be required for photoperiodic flowering. To our knowledge, this is the first systematic investigation on the lncRNAs involved in NB-responses of quinoa. In total, we identified 17 putative positiveand 7 putative negative-flowering lncRNAs. Coexpression network analysis indicated that 15 positiveflowering lncRNAs may compete with 5 negativeflowering lncRNAs to control a few pivotal flowering homologous genes, which might be the basis of NB responses in quinoa. Collectively, these results will deepen our understanding of the roles of lncRNAs in flowering, and provide valuable genetic resources for yield improvement of quinoa.

\section{Methods}

\section{Plant materials and growth conditions}

Quinoa (Chenopodium quinoa) cultivar "HL1" and "HL2" were used for flowering time analysis. The two cultivars have nearly the same floral transition time, however, "HL2" needs longer time (about 115 d) compared with "HL1" (about $60 \mathrm{~d}$ ) for post-flowering development. Seeds were harvested from the Experimental Station of Key Laboratory of Coarse Cereal Processing, Ministry of Agriculture and Rural Affairs, in 2019 at Liangshan, Sichuan province. Seeds were sown in a pot with $20 \mathrm{~cm}$ in diameter and $18 \mathrm{~cm}$ in depth. Quinoa seedlings were maintained for $14 \mathrm{~d}$ in a greenhouse at Chengdu University under natural day conditions. Then, the seedlings pots were transferred into growth chambers (Jiang Nan, Ning Bo, China) subjecting to SD and NB treatment. The floral buds developmental situation of both cultivers under different conditions was observed every day.

For RNA-seq, the seeds of "HL1" were sown in pots with the same size as described above. After sowing, the pots were placed in growth chambers for SD treatment with daily cycles of $8 \mathrm{~h}$ light at $25^{\circ} \mathrm{C}$ and $16 \mathrm{~h}$ dark at $23{ }^{\circ} \mathrm{C}$. The relative humidity was $70 \%$, and light intensity was $750 \mu \mathrm{mol} . \mathrm{m}^{-2} \cdot \mathrm{s}^{-1}$. On 7 DAS, 15 robust shoots were retained in each pot by removing the spared ones. On 14 DAS (SD1) and 26 DAS (SD2), the top two fully expanded leaves of each seedling were collected at $2 \mathrm{~h}$ after dawn, respectively. Then, the growth conditions were reset to night-break conditions (NB) with daily cycles of 8 $\mathrm{h}$ light at $25^{\circ} \mathrm{C}$ and $7 \mathrm{~h}$ dark, $1 \mathrm{~h}$ light exposure and $8 \mathrm{~h}$ dark at $23^{\circ} \mathrm{C}$. After $2 \mathrm{~d}$ NB treatment, namely on 28 DAS, the leaf samples were collected as above. Sampling was performed with three replicates and each replicate contains 5 individual plants. The harvested fresh leaf samples were immediately frozen by liquid nitrogen and stored at $-80^{\circ} \mathrm{C}$ before RNA extraction.

\section{Construction of strand-specific RNA sequencing libraries}

Total RNA was extracted from each leaves sample using Trizol reagent (Invitrogen, CA, USA) according to the manufacturer's instructions. RNA degradation was checked by $1.5 \%$ agarose gel electrophoresis. RNA concentration and purity of each sample were measured to be qualified for downstream experiments. Only the samples with RIN (RNA Integrity Number) value larger than 7.5 were used for long-noncoding RNA sequencing libraries construction. An amount of $1.5 \mu \mathrm{g}$ total RNA per sample was input for rRNA removal reaction by using the Ribo-Zero rRNA Removal Kit (Epicentre, Madison, WI, USA). Then, strand-specific RNA sequencing libraries were generated using NEBNext ${ }^{\mathrm{R}}$ Ultra $^{\mathrm{Tw}}$ Directional RNA Library Prep Kit for Illumina ${ }^{\mathrm{R}}$ (NEB, USA) according to manufacturer's protocol.

\section{Data analysis}

RNA sequencing was performed on an Illumina platform (Hiseq 4000) to generate 150 bp paired-end reads. Raw reads from 9 libraries were filtered by removing the unqualified reads (adapter-, ploy-N-containing and lowquality reads) to obtain clean reads. By applying HISAT2 software [38], clean reads were mapped to the quinoa reference genome sequences (https://www.cbrc.kaust. edu.sa/chenopodiumdb) [37]. Based on the mapping results, StringTie [39] was used to re-construct the transcripts. Gffcompare program [40] was used to annotate the assembled transcripts, and the unannotated transcripts were further used for long-noncoding RNA prediction. Transcripts with lengths $>200 \mathrm{bp}$ and exon number $\geq 2$ were retained as IncRNA candidates whose protein-coding potential were further evaluated by using CPC (Coding Potential Calculator) [63], CNCI (CodingNon-Coding Index) [64], CPAT (Coding Potential Assessment Tool) [65] and Pfam programs [66] to obtain the final lncRNAs. LncRNAs were categorized into different types of lncRNAs including lincRNA, intronic 
lncRNA, anti-sense lncRNA and sense lncRNA by using Cuffcompare program.

\section{Differential expression analysis}

The expression levels of both mRNA and lncRNA genes were calculated and normalized to FPKM (fragments per kilo-base of exon per million fragments mapped) [42] value using StringTie [39]. Differentially expressed genes (DEGs) between different groups were determined by using DESeq2 $\mathrm{R}$ package [43] based on the negative binomial distribution model. The resulting $P$ values were adjusted using the Benjamini and Hochberg's approach for controlling the false discovery rate (FDR). Thresholds of an FDR $<0.05$ and $\mid \log 2$ (fold change) $\mid \geq 1$ were applied to call DEGs.

\section{Target gene prediction}

For cis targets identification, the protein-coding genes located at $100 \mathrm{~kb}$ upstream and downstream of lncRNA were selected and functionally annotated. To identify the trans targets, the correlation between mRNAs and lncRNAs was evaluated using the Pearson's correlation coefficient (PCC) based on their expression profiles at different samples. mRNA-lncRNA pairs with |PCC value $\mid>0.9$ and $P$ value $<0.01$ were designated as coexpressed gene pairs in which the mRNA genes were identified as the trans targets of lncRNAs.

\section{Functional enrichment analysis of the targets of differentially expressed IncRNAs}

To understand the biological function of differentially expressed lncRNAs (DE IncRNAs), Kyoto Encyclopedia of Genes and Genomes (KEGG) [67] and Gene Ontology enrichment analysis [68] of the corresponding target genes was conducted. KEGG enrichment analysis was performed by using KOBAS software and the pathways with corrected $P$ value less than 0.05 were taken as significantly enriched by the target genes. GO enrichment analysis of the target genes was implemented by the topGO R packages. The most significantly enriched GO terms were selected with the cutoff of corrected $P$ value $<0.05$.

\section{Co-expression network analysis and visualization}

Based on the expression data, the PCC between lncRNAs and mRNAs was obtained. Co-expressed lncRNA-mRNA pairs were visualized using Cytoscape (Version 3.7.2) [69]. By using the Cytoscape tools "Network Analyzer-Network analysis", the network was analyzed and the degrees of nodes were obtained.

\section{Abbreviations}

SD: Short-day; NB: Night-break; IncRNAs: Long noncoding RNAs; FPKM: Fragments Per Kilobase of transcript sequence per Millions base pairs sequenced; SDP: Short-day plant; LDP: Long-day plant; DAS: Days after sowing; GO: Gene ontology; KEGG: Kyoto Encyclopedia of Genes and Genomes; PCC: Pearson's correlation coefficient; CPC: Coding Potential Calculator; CNCl: Coding-Non-Coding Index; CPAT: Coding Potential Assessment Tool

\section{Supplementary Information}

The online version contains supplementary material available at https://doi. org/10.1186/s12864-021-07605-2.

Additional file 1: Fig. S1. Spearman correlation coefficients between different samples

Additional file 2: Table S1. Expression levels of all DE IncRNAs

Additional file 3: Table S2. Expression levels of all DEGs

Additional file 4: Table S3. Targets of the up-regulated IncRNAs in SD1_vs_SD2

Additional file 5: Table S4. Targets of the down-regulated IncRNAs in SD1_vs_SD2

Additional file 6: Table S5. Targets of the up-regulated IncRNAs in SD2_vs_NB

Additional file 7: Table S6. Targets of the down-regulated IncRNAs in SD2_vs_NB

Additional file 8: Table S7. Network of the 17 positive flowering IncRNAs

Additional file 9: Table S8. Network of the 7 negative flowering IncRNAs

Authors' contributions

QW conceived and designed this study. QW wrote the manuscript. QW, YL and $X W$ performed most of the experiments. $X Y, C L, X B$ and $Q L$ participated in analyzing the RNA-seq data. YL, DX and YW prepared the samples for sequencing. $L Z$ and $G Z$ participated in quinoa flowering phenotype analysis. All authors have read and approved the final manuscript.

Funding

This work is supported by the National Natural Science Foundation of China (Grant 31701493); the Sichuan Science and Technology Program (Grant 2018JY0344, Grant 2020YJ0199); the China Agriculture Research System (Project CARS-08-02A). The funding bodies have no role in the design of the study and collection, analysis, and interpretation of data and in writing the manuscript.

Availability of data and materials

The transcriptome raw data produced in this study is deposited at NCBI SRA database (http://trace.ncbi.nlm.nih.gov/Traces/sra) under accession PRJNA673052.

\section{Declarations}

Ethics approval and consent to participate

The plant seeds used in this study were harvested from the Experimental Station of Key Laboratory of Coarse Cereal Processing, Ministry of Agriculture and Rural Affairs. The plants were grown in green house and controlled growth chamber, and all the experiments were carried out in Key Laboratory of Coarse Cereal Processing, Ministry of Agriculture and Rural Affairs, Chengdu University. All the Experimental research and field studies, including the collection of plant material, comply with the guidelines of Key Laboratory of Coarse Cereal Processing, Ministry of Agriculture and Rural Affairs, Chengdu University.

Consent for publication

Not applicable.

Competing interests

The authors declare that they have no competing interests. 


\section{Received: 4 December 2020 Accepted: 8 April 2021}

\section{Published online: 19 April 2021}

\section{References}

1. Fornara F, de Montaigu A, Coupland G. SnapShot: Control of flowering in Arabidopsis. Cell. 2010;141(3):550, 550.e551-2.

2. Wigge PA. FT, a mobile developmental signal in plants. Curr Biol. 2011;21(9): R374-8. https://doi.org/10.1016/j.cub.2011.03.038.

3. Andres F, Coupland $G$. The genetic basis of flowering responses to seasonal cues. Nat Rev Genet. 2012;13(9):627-39. https://doi.org/10.1038/nrg3291.

4. Song $\mathrm{YH}$, Ito $\mathrm{S}$, Imaizumi T. Flowering time regulation: photoperiod- and temperature-sensing in leaves. Trends Plant Sci. 2013;18(10):575-83. https:// doi.org/10.1016/j.tplants.2013.05.003.

5. Taoka K, Ohki I, Tsuji H, Furuita K, Hayashi K, Yanase T, et al. 14-3-3 proteins act as intracellular receptors for rice Hd3a florigen. Nature. 2011;476(7360): 332-5. https://doi.org/10.1038/nature10272.

6. Putterill J, Varkonyi-Gasic E. FT and florigen long-distance flowering control in plants. Curr Opin Plant Biol. 2016;33:77-82. https://doi.org/10.1016/j.pbi.2 016.06.008.

7. Itoh $\mathrm{H}$, Nonoue $\mathrm{Y}$, Yano $\mathrm{M}$, Izawa $\mathrm{T}$. A pair of floral regulators sets critical day length for Hd3a florigen expression in rice. Nat Genet. 2010;42(7):635-8. https://doi.org/10.1038/ng.606.

8. Kong F, Liu B, Xia Z, Sato S, Kim BM, Watanabe S, et al. Two coordinately regulated homologs of FLOWERING LOCUS T are involved in the control of photoperiodic flowering in soybean. Plant Physiol. 2010;154(3):1220-31. https://doi.org/10.1104/pp.110.160796.

9. Shimada S, Ogawa T, Kitagawa S, Suzuki T, Ikari C, Shitsukawa N, et al. A genetic network of flowering-time genes in wheat leaves, in which an APET ALA1/FRUITFULL-like gene, VRN1, is upstream of FLOWERING LOCUS T. Plant J. 2009;58(4):668-81. https://doi.org/10.1111/j.1365-313X.2009.03806.x.

10. Corbesier L, Vincent C, Jang S, Fornara F, Fan Q, Searle I, et al. FT protein movement contributes to long-distance signaling in floral induction of Arabidopsis. Science. 2007;316(5827):1030-3. https://doi.org/10.1126/ science.1141752

11. Ishikawa R, Tamaki S, Yokoi S, Inagaki N, Shinomura T, Takano M, et al. Suppression of the floral activator $\mathrm{Hd} 3 \mathrm{a}$ is the principal cause of the night break effect in rice. Plant Cell. 2005;17(12):3326-36. https://doi.org/10.1105/ tpc.105.037028.

12. Xu M, Yamagishi N, Zhao C, Takeshima R, Kasai M, Watanabe S, et al. The soybean-specific maturity gene E1 family of floral repressors controls nightbreak responses through Down-regulation of FLOWERING LOCUS T Orthologs. Plant Physiol. 2015;168(4):1735-46. https://doi.org/10.1104/pp.15. 00763.

13. Pearce S, Shaw LM, Lin H, Cotter JD, Li C, Dubcovsky J. Night-break experiments shed light on the Photoperiod1-mediated flowering. Plant Physiol. 2017;174(2):1139-50. https://doi.org/10.1104/pp.17.00361.

14. Ishikawa R, Shinomura T, Takano M, Shimamoto K. Phytochrome dependent quantitative control of $\mathrm{Hd} 3 \mathrm{a}$ transcription is the basis of the night break effect in rice flowering. Genes Genet Syst. 2009:84(2):179-84. https://doi. org/10.1266/ggs.84.179.

15. Ramos-Sánchez JM, Triozzi PM, Alique D, Geng F, Gao M, Jaeger KE, et al. LHY2 Integrates Night-Length Information to Determine Timing of Poplar Photoperiodic Growth. Curr Biol. 2019;29(14):2402-2406.e2404.

16. Chekanova JA. Long non-coding RNAs and their functions in plants. Curr Opin Plant Biol. 2015;27:207-16. https://doi.org/10.1016/j.pbi.2015.08.003.

17. Liu X, Hao L, Li D, Zhu L, Hu S. Long non-coding RNAs and their biological roles in plants. Genomics Proteomics Bioinformatics. 2015;13(3):137-47. https://doi.org/10.1016/j.gpb.2015.02.003.

18. Hu S, Wang X, Shan G. Insertion of an Alu element in a IncRNA leads to primate-specific modulation of alternative splicing. Nat Struct Mol Biol. 2016; 23(11):1011-9. https://doi.org/10.1038/nsmb.3302.

19. Wei S, Chen H, Dzakah EE, Yu B, Wang X, Fu T, et al. Systematic evaluation of C. elegans lincRNAs with CRISPR knockout mutants. Genome Biol. 2019; 20(1):7.

20. Rashid F, Shah A, Shan G. Long non-coding RNAs in the cytoplasm. Genomics Proteomics Bioinformatics. 2016;14(2):73-80. https://doi.org/10.1 016/j.gpb.2016.03.005.

21. Yu B, Shan G. Functions of long noncoding RNAs in the nucleus. Nucleus. 2016;7(2):155-66. https://doi.org/10.1080/19491034.2016.1179408.
22. Kim DH, Xi Y, Sung S. Modular function of long noncoding RNA, COLDAIR, in the vernalization response. PLoS Genet. 2017;13(7):e1006939. https://doi. org/10.1371/journal.pgen.1006939.

23. Heo JB, Sung S. Vernalization-mediated epigenetic silencing by a long intronic noncoding RNA. Science. 2011;331(6013):76-9. https://doi.org/1 $0.1126 /$ science.1197349.

24. Sun Q, Csorba T, Skourti-Stathaki K, Proudfoot NJ, Dean C. R-loop stabilization represses antisense transcription at the Arabidopsis FLC locus. Science. 2013;340(6132):619-21. https://doi.org/10.1126/science.1234848.

25. Ding J, Lu Q, Ouyang Y, Mao H, Zhang P, Yao J, et al. A long noncoding RNA regulates photoperiod-sensitive male sterility, an essential component of hybrid rice. Proc Natl Acad Sci U S A. 2012;109(7):2654-9. https://doi. org/10.1073/pnas.1121374109.

26. Franco-Zorrilla JM, Valli A, Todesco M, Mateos I, Puga MI, Rubio-Somoza I, et al. Target mimicry provides a new mechanism for regulation of microRNA activity. Nat Genet. 2007;39(8):1033-7. https://doi.org/10.1038/ng2 079.

27. Liu X, Li D, Zhang D, Yin D, Zhao Y, Ji C, et al. A novel antisense long noncoding RNA, TWISTED LEAF, maintains LEAF blade flattening by regulating its associated sense R2R3-MYB gene in rice. New Phytol. 2018; 218(2):774-88. https://doi.org/10.1111/nph.15023.

28. Campalans A, Kondorosi A, Crespi M. Enod40, a short open reading framecontaining mRNA, induces cytoplasmic localization of a nuclear RNA binding protein in Medicago truncatula. Plant Cell. 2004;16(4):1047-59. https://doi.org/10.1105/tpc.019406.

29. Ariel F, Jegu T, Latrasse D, Romero-Barrios N, Christ A, Benhamed M, et al. Noncoding transcription by alternative RNA polymerases dynamically regulates an auxin-driven chromatin loop. Mol Cell. 2014;55(3):383-96. https://doi.org/10.1016/j.molcel.2014.06.011.

30. Huang L, Dong H, Zhou D, Li M, Liu Y, Zhang F, et al. Systematic identification of long non-coding RNAs during pollen development and fertilization in Brassica rapa. Plant J. 2018;96(1):203-22. https://doi.org/1 0.1111/tpj.14016.

31. Tian Y, Bai S, Dang Z, Hao J, Zhang J, Hasi A. Genome-wide identification and characterization of long non-coding RNAs involved in fruit ripening and the climacteric in Cucumis melo. BMC Plant Biol. 2019;19(1):369. https:// doi.org/10.1186/s12870-019-1942-4.

32. Fu L, Ding Z, Tan D, Han B, Sun X, Zhang J. Genome-wide discovery and functional prediction of salt-responsive IncRNAs in duckweed. BMC Genomics. 2020;21(1):212.

33. Tan X, Li S, Hu L, Zhang C. Genome-wide analysis of long non-coding RNAs (IncRNAs) in two contrasting rapeseed (Brassica napus L.) genotypes subjected to drought stress and re-watering. BMC Plant Biol. 2020;20(1):81.

34. Lopez-Marques RL, Norrevang AF, Ache P, Moog M, Visintainer D, Wendt T, et al. Prospects for the accelerated improvement of the resilient crop quinoa. J Exp Bot. 2020;71(18):5333-47. https://doi.org/10.1093/jxb/eraa285.

35. Storchova H, Hubackova H, Abeyawardana OAJ, Walterova J, Vondrakova Z, Eliasova $\mathrm{K}$, et al. Chenopodium ficifolium flowers under long days without upregulation of FLOWERING LOCUS T (FT) homologs. Planta. 2019;250(6): 2111-25. https://doi.org/10.1007/s00425-019-03285-1

36. Christiansen JL, Jacobsen SE, Jørgensen ST. Photoperiodic effect on flowering and seed development in quinoa (Chenopodium quinoaWilld.). Acta Agric Scand B Soil Plant Sci. 2010;60(6):539-44. https://doi.org/10.1080/ 09064710903295184

37. Jarvis DE, Ho YS, Lightfoot DJ, Schmockel SM, Li B, Borm TJ, et al. The genome of Chenopodium quinoa. Nature. 2017;542(7641):307-12. https:// doi.org/10.1038/nature21370

38. Kim D, Langmead B, Salzberg SL. HISAT: a fast spliced aligner with low memory requirements. Nat Methods. 2015;12(4):357-60. https://doi.org/10.1 038/nmeth.3317.

39. Pertea M, Pertea GM, Antonescu CM, Chang TC, Mendell JT, Salzberg SL. StringTie enables improved reconstruction of a transcriptome from RNA-seq reads. Nat Biotechnol. 2015;33(3):290-5. https://doi.org/10.1038/nbt.3122.

40. Pertea G, Pertea M. GFF Utilities: GffRead and GffCompare. F1000Res. 2020;9: ISCB Comm J-304.

41. Liu C, Bai B, Skogerbo G, Cai L, Deng W, Zhang Y, et al. NONCODE: an integrated knowledge database of non-coding RNAs. Nucleic Acids Res. 2005:33(Database issue):D112-5. https://doi.org/10.1093/nar/gki041.

42. Trapnell C, Williams BA, Pertea G, Mortazavi A, Kwan G, van Baren MJ, et al. Transcript assembly and quantification by RNA-Seq reveals unannotated 
transcripts and isoform switching during cell differentiation. Nat Biotechnol. 2010;28(5):511-5. https://doi.org/10.1038/nbt.1621.

43. Love Ml, Huber W, Anders S. Moderated estimation of fold change and dispersion for RNA-seq data with DESeq2. Genome Biol. 2014;15(12):550. https://doi.org/10.1186/s13059-014-0550-8.

44. Luccioni L, Krzymuski M, Sanchez-Lamas M, Karayekov E, Cerdan PD, Casal J. CONSTANS delays Arabidopsis flowering under short days. Plant J. 2019; 97(5):923-32. https://doi.org/10.1111/tpj.14171.

45. Yang Y, Peng Q, Chen GX, Li XH, Wu CY. OsELF3 is involved in circadian clock regulation for promoting flowering under long-day conditions in rice. Mol Plant. 2013;6:202-15. https://doi.org/10.1093/mp/sss062.

46. Zhao J, Huang X, Ouyang X, Chen W, Du A, Zhu L, et al. OsELF3-1, an Ortholog of Arabidopsis EARLY FLOWERING 3, regulates Rice circadian rhythm and photoperiodic flowering. PLoS One. 2012;7(8):e43705. https:// doi.org/10.1371/journal.pone.0043705.

47. Alabadi D, Yanovsky MJ, Mas P, Harmer SL, Kay SA. Critical role for CCA1 and LHY in maintaining circadian rhythmicity in Arabidopsis. Curr Biol. 2002; 12(9):757-61. https://doi.org/10.1016/50960-9822(02)00815-1.

48. Hajdu A, Dobos O, Domijan M, Balint B, Nagy I, Nagy F, et al. ELONGATED HYPOCOTYL 5 mediates blue light signalling to the Arabidopsis circadian clock. Plant J. 2018;96(6):1242-54. https://doi.org/10.1111/tpj.14106.

49. Osnato M, Matias-Hernandez L, Aguilar-Jaramillo AE, Kater MM, Pelaz S Genes of the RAV family control heading date and carpel development in Rice. Plant Physiol. 2020;183(4):1663-80. https://doi.org/10.1104/pp.20.00562.

50. Neff MM, Chory J. Genetic interactions between phytochrome a, phytochrome B, and cryptochrome 1 during Arabidopsis development. Plant Physiol. 1998;1 18(1):27-35. https://doi.org/10.1104/pp.118.1.27.

51. Fang J, Zhang F, Wang H, Wang W, Zhao F, Li Z, et al. Ef-cd locus shortens rice maturity duration without yield penalty. Proc Natl Acad Sci U S A. 2019; 116(37):18717-22. https://doi.org/10.1073/pnas.1815030116.

52. Bazile $D$, Jacobsen $S E$, Verniau A. The global expansion of quinoa: trends and limits. Front Plant Sci. 2016;7:622.

53. Yang Z, Yang C, Wang Z, Yang Z, Chen D, Wu Y. LncRNA expression profile and ceRNA analysis in tomato during flowering. PLoS One. 2019;14(1): e0210650. https://doi.org/10.1371/journal.pone.0210650.

54. Ding Z, Tie W, Fu L, Yan Y, Liu G, Yan W, et al. Strand-specific RNA-sea based identification and functional prediction of drought-responsive IncRNAs in cassava. BMC Genomics. 2019;20(1):214. https://doi.org/10.1186/ s12864-019-5585-5.

55. Ma X, Zhang X, Traore SM, Xin Z, Ning L, Li K, Zhao K, Li Z, He G, Yin D: Genome-wide identification and analysis of long noncoding RNAs (IncRNAs) during seed development in peanut (Arachis hypogaea L.). BMC Plant Biol 2020, 20(1):192.

56. Wickland DP, Hanzawa Y. The FLOWERING LOCUS T/TERMINAL FLOWER 1 gene family: functional evolution and molecular mechanisms. Mol Plant. 2015;8(7):983-97. https://doi.org/10.1016/j.molp.2015.01.007.

57. Komiya R, Ikegami A, Tamaki S, Yokoi S, Shimamoto K. Hd3a and RFT1 are essential for flowering in rice. Development. 2008;135(4):767-74. https://doi. org/10.1242/dev.008631.

58. Liu W, Jiang B, Ma L, Zhang S, Zhai H, Xu X, et al. Functional diversification of flowering locus T homologs in soybean: GmFT1a and GmFT2a/5a have opposite roles in controlling flowering and maturation. New Phytol. 2018; 217:1335-45. https://doi.org/10.1111/nph.14884.

59. Chab D, Kolar J, Olson MS, Storchova H. Two flowering locus T (FT) homologs in Chenopodium rubrum differ in expression patterns. Planta. 2008;228(6):929-40. https://doi.org/10.1007/s00425-008-0792-3.

60. Golicz AA, Steinfort U, Arya H, Singh MB, Bhalla PL. Analysis of the quinoa genome reveals conservation and divergence of the flowering pathways. Funct Integr Genomics. 2020;20(2):245-58. https://doi.org/10.1007/s10142-01 9-00711-1.

61. Tsuji H. Taoka K-i, Shimamoto K: Florigen in rice: complex gene network for florigen transcription, florigen activation complex, and multiple functions. Curr Opin Plant Biol. 2013;16(2):228-35. https://doi.org/10.1016/.jpbi.2013.01. 005.

62. Higuchi $Y$, Sumitomo $K$, Oda A, Shimizu H, Hisamatsu T. Day light quality affects the night-break response in the short-day plant chrysanthemum, suggesting differential phytochrome-mediated regulation of flowering. J Plant Physiol. 2012;169(18):1789-96. https://doi.org/10.1016/j.jplph.2012.07. 003.
63. Kong L, Zhang Y, Ye ZQ, Liu XQ, Zhao SQ, Wei L, et al. CPC: assess the protein-coding potential of transcripts using sequence features and support vector machine. Nucleic Acids Res. 2007;35(Web Server issue):W345-9.

64. Sun L, Luo H, Bu D, Zhao G, Yu K, Zhang C, et al. Utilizing sequence intrinsic composition to classify protein-coding and long non-coding transcripts. Nucleic Acids Res. 2013;41(17):e166. https://doi.org/10.1093/nar/gkt646.

65. Wang L, Park HJ, Dasari S, Wang S, Kocher JP, Li W. CPAT: coding-potential assessment tool using an alignment-free logistic regression model. Nucleic Acids Res. 2013;41(6):e74. https://doi.org/10.1093/nar/gkt006.

66. Finn RD, Coggill P, Eberhardt RY, Eddy SR, Mistry J, Mitchell AL, et al. The Pfam protein families database: towards a more sustainable future. Nucleic Acids Res. 2016;44(D1):D279-85. https://doi.org/10.1093/nar/gkv1344.

67. Kanehisa M, Goto S. KEGG: Kyoto encyclopedia of genes and genomes. Nucleic Acids Res. 2000;28(1):27-30. https://doi.org/10.1093/nar/28.1.27.

68. Ashburner M, Ball CA, Blake JA, Botstein D, Butler H, Cherry JM, et al. Gene ontology: tool for the unification of biology. The gene ontology consortium. Nat Genet. 2000;25(1):25-9. https://doi.org/10.1038/75556.

69. Shannon P, Markiel A, Ozier O, Baliga NS, Wang JT, Ramage D, et al. Cytoscape: a software environment for integrated models of biomolecular interaction networks. Genome Res. 2003;13(11):2498-504. https://doi.org/1 $0.1101 /$ gr.1239303

\section{Publisher's Note}

Springer Nature remains neutral with regard to jurisdictional claims in published maps and institutional affiliations.
Ready to submit your research? Choose BMC and benefit from:

- fast, convenient online submission

- thorough peer review by experienced researchers in your field

- rapid publication on acceptance

- support for research data, including large and complex data types

- gold Open Access which fosters wider collaboration and increased citations

- maximum visibility for your research: over $100 \mathrm{M}$ website views per year

At BMC, research is always in progress.

Learn more biomedcentral.com/submissions 